\title{
Reducible Gauge Theories in Local Superfield Lagrangian BRST Quantization
}

\author{
D. M. Gitman ${ }^{a)}$, P.Yu. Moshin ${ }^{a), b)}$, and A.A. Reshetnyak ${ }^{c)}$ \\ a) Instituto de Física, Universidade de São Paulo, \\ Caixa Postal 66318-CEP, 05315-970 São Paulo, S.P., Brazil \\ b) Tomsk State Pedagogical University, 634041 Tomsk, Russia \\ c) Laboratory of Non-equilibrium State Theory, Institute of \\ Strength Physics and Materials Science, 634021 Tomsk, Russia
}

Received on 29 August, 2007

\begin{abstract}
The construction of $\theta$-local superfield Lagrangian BRST quantization in non-Abelian hypergauges for generic gauge theories based on the action principle is examined in the case of reducible local superfield models (LSM) on the basis of embedding a gauge theory into a special $\theta$-local superfield model with antisymplectic constraints and a Grassmann-odd time parameter $\theta$. We examine the problem of establishing a new correspondence between the odd-Lagrangian and odd-Hamiltonian formulations of a local LSM in the case of degeneracy of the Lagrangian description with respect to derivatives over $\theta$ of generalized classical superfields $\mathcal{A}^{I}(\theta)$. We also reveal the role of the nilpotent BRST-BFV charge for a formal dynamical system corresponding to the BV-BFV dual description of an LSM.
\end{abstract}

Keywords: Lagrangian (superfield) BV quantization; Odd Lagrangian and Hamiltonian formulations; Second-class constraints; Reducible multi-level non-Abelian hypergauges

\section{INTRODUCTION}

Local superfield Lagrangian BRST quantization [1] was originally proposed for irreducible gauge theories and Abelian hypergauges [2], which introduce the gauge-fixing procedure following the BV method [3], and then extended to arbitrary gauge models in reducible non-Abelian hypergauges of finite stage of reducibility [4]. The quantization rules [1] combine, in terms of superfields, a generalization of the "firstlevel" Batalin-Tyutin formalism [5] (the case of reducible hypergauges is examined in [6]) and a geometric realization of BRST transformations $[7,8]$ in the particular case of $\theta$-local superfield models (LSM) of Yang-Mills-type. The concept of an $\operatorname{LSM}[1,2,4]$, which realizes a trivial relation between the even $t$ and odd $\theta$ components of the object $\chi=(t, \theta)$ called supertime [9], unlike the nontrivial interrelation realized by the operator $D=\partial_{\theta}+\theta \partial_{t}$ in the Hamiltonian superfield $N=1$ formalism [10] of the BFV quantization [11], provides the basis for the method of local quantization $[1,2,4]$ and proves to be fruitful in solving a number problems that restrict the applicability of the functional superfield Lagrangian method [12] to specific gauge theories. The idea of an LSM makes it possible to obtain an odd-Lagrangian and odd-Hamiltonian form of the classical master equation as a condition that preserves a $\theta$-local analogue of the energy by virtue of Noether's first theorem with respect to the evolution along the variable $\theta$, defined by superfield extensions of the extremals for an initial gauge model, i.e., by odd-Lagrangian (LS) and oddHamiltonian (HS) systems. The concept of an LSM provides an inclusion of the dual BV-BFV description [13, 14] of a reducible gauge theory in terms of a BRST charge for formal topological dynamical systems (i.e., systems without a definite time parameter) subject to first-class constraints of higher-stage reducibility in the problem of embedding a gauge algebra of a special reducible LSM into that of a general[25] LSM. Finally, the idea of an LSM proves to be an adequate extension of a usual gauge model in a superfield construction of the quantum action as a superfield analogue of the KoszulTate complex resolution $[15,16]$ in Lagrangian formalism on the basis of interpreting the reducibility relations as special gauge transformations of ghosts transformed into a unique $\theta$ integrable odd HS.

Along the lines of our previous works, we consider it an interesting task to solve two of the problems mentioned in the conclusion of the first paper of Ref. [1]. The first problem is that of establishing a different (from the one given by a Legendre transformation $[1,2,4]$ ) correspondence between the oddLagrangian and odd-Hamiltonian formulations of an LSM in the case of degeneracy of the Lagrangian description with respect to derivatives over $\theta$ of generalized classical superfields $\mathcal{A}^{I}(\theta)$, i.e., $\overleftarrow{\partial}_{\theta} \mathcal{A}^{I}(\theta)$. The second problem is to reveal the role of the nilpotent BRST-BFV charge for a formal dynamical system corresponding to the BV-BFV dual description of an LSM.

We devote this paper to the solution of the following problems:

1. A construction of an odd-Hamiltonian formulation for an LSM starting from an odd Lagrangian in the case of a degenerate Hessian supermatrix $\left\|\left(S_{\mathrm{L}}^{\prime \prime}\right)_{I J}\right\|(\theta)$ of Refs. $[1,2,4]$ as the supermatrix of second derivatives of the Lagrangian classical action, $S_{\mathrm{L}}(\theta)=$ $S_{\mathrm{L}}\left(\mathcal{A}(\theta), \partial_{\theta} \mathcal{A}(\theta), \theta\right)$, with respect to odd velocities $\left(\partial_{\theta} \mathcal{A}^{I}, \partial_{\theta} \mathcal{A}^{J}\right)(\theta)$ on the basis of Dirac's algorithm in terms of a $\theta$-local antibracket.

2. An application of the BFV method [11] to a construction of formal counterparts of the BFV-BRST charge, gauge fermion and unitarizing Hamiltonian of a $t$-local field theory in terms of a $\theta$-local Dirac's antibracket, reflecting, in view of general gauge invariance, the presence of a subsystem of second-class constraints among all of the antisymplectic constraints. 
3. Establishing a correspondence between the resulting odd-Hamiltonian formulation of an LSM with the BV quantum action for the gauge model corresponding to an LSM.

The paper is organized as follows. In Section 2, we apply Dirac's algorithm [17] to realize an odd Hamiltonization of a Lagrangian degenerate LSM, being an extension of a usual model of classical fields $A^{i}, i=1, \ldots, n=n_{+}+n_{-}$, on a configuration space $M_{\mathrm{cl}}$, to a $\theta$-local theory defined on an odd tangent bundle $T_{\text {odd }} \mathcal{M}_{\mathrm{CL}} \equiv \Pi T \mathcal{M}_{\mathrm{CL}}=\left\{\mathcal{A}^{I}, \partial_{\theta} \mathcal{A}^{I}\right\}$, $I=1, \ldots, N=N_{+}+N_{-},\left(n_{+}, n_{-}\right) \leq\left(N_{+}, N_{-}\right)$with $N_{+},\left(N_{-}\right)$ bosonic (fermionic) superfields. The generalized classical superfields $\mathcal{A}^{I}(\theta), \mathcal{A}^{I}(\theta)=A^{I}+\lambda^{I} \theta$, parameterize the base $\mathcal{M}_{\mathrm{CL}}$ $\left(\mathcal{M}_{\mathrm{cl}} \subset \mathcal{M}_{\mathrm{CL}}\right)$ of the bundle $\Pi T \mathcal{M}_{\mathrm{CL}}$ and transform with respect to a $J$-superfield representation $T$ of the direct product of supergroups $\bar{J}, P: J=\bar{J} \times P, P=\exp \left(i \mu p_{\theta}\right)[1,2,4]$, with $\bar{J}$ chosen as a spacetime SUSY group[26], and $\mu, p_{\theta}$ being the respective nilpotent parameter and generator of $\theta$ shifts. The non-Lorentz [18] character of superfields $\mathcal{A}^{I}(\theta)$ defined on $\mathcal{M}=\left\{\left(z^{M}, \theta\right)\right\}=\left\{z^{K}\right\}, z^{M} \subset i \subset I$, is reflected by a possible inclusion in their spectrum of additional (besides $\mathcal{A}^{i}(\theta)$ ) superfields corresponding to the ghosts of the minimal sector in the BV quantization scheme [3]. In Section 3, following the BFV prescription, we construct $\theta$-local counterparts of the ghosts on the basis of a complete system of antisymplectic constraints and a Hamiltonian action $S_{\mathrm{H}_{0}}(\theta)$ defined on $\Pi T^{*} \mathcal{M}_{\mathrm{CL}}=\left\{\mathcal{A}^{I}, \mathcal{A}_{I}^{*}\right\}$, a bosonic BRST charge $S_{\Omega}(\theta)$, a unitarizing Hamiltonian action $S_{\mathcal{H}}(\theta)$, and a gauge fermion $F_{\Psi}(\theta)$. We specify to the case of a singular LSM a derivation of Lagrangian and Hamiltonian master equations from Noether's first theorem [19] applied to $\theta$-shifts, and establish a relation between the complete Hamiltonian action, $S_{\mathrm{H}}(\theta)=S_{\mathcal{H}}(\theta)+\left(S_{\Omega}(\theta), F_{\Psi}(\theta)\right)_{\theta D}$, constructed via $\theta$-local Dirac's antibracket, and the quantum action of the BV method [3].

We mostly follow the conventions of Refs. [1, 4] based on DeWitt's condensed notation [20] and distinguish between two types of superfield derivatives: the right (left) derivative $\overleftarrow{\partial} \mathcal{F}(\theta) / \partial \Gamma^{p}(\theta)\left(\vec{\partial} \mathcal{F}(\theta) / \partial \Gamma^{p}(\theta)\right)$ of a function $\mathcal{F}(\theta)$ for a fixed $\theta$, and the right (left) variational derivative $\overleftarrow{\delta} F / \delta \Phi^{A}(\theta)$ $\left(\vec{\delta} F / \delta \Phi^{A}(\theta)\right)$ of a functional $F$. In the same manner, superfield right (left) covariant derivatives with respect to a superfield $\Gamma^{p}(\theta)$ are denoted by $\overleftarrow{\nabla}_{p}(\theta) F\left(\vec{\nabla}_{p}(\theta) F\right)$ for a fixed $\theta$, and variational derivatives are denoted by $\overleftarrow{\mathcal{D}}_{p}(\theta) F$
$\left(\overrightarrow{\mathcal{D}}_{p}(\theta) F\right)$. Derivatives with respect to super(anti)fields and their components are understood as acting from the right (left), for instance, $\delta / \delta \lambda_{A}^{*}$ or $\delta / \delta \Phi_{A}(\theta)$; in the opposite case we use arrows “ $\rightarrow$ " (“ $\longleftarrow$ ”) for left (right) differentiation. For right-hand derivatives with respect to $\mathcal{A}^{I}(\theta)$ for a fixed $\theta$, we use the notation $\mathcal{F}{ }_{I}(\theta) \equiv \partial \mathcal{F}(\theta) / \partial \mathcal{A}^{I}(\theta)$. As in [1, 4], following the definitions of Refs. [21, 22], a smooth supersurface [27] $\Sigma$, is parameterized by local coordinates $z^{i}(\theta)$, and the rank of an even $\theta$-local supermatrix $\|G(\theta)\|$ with $Z_{2}$ grading $\varepsilon$ is characterized by a pair of numbers $\bar{m}=\left(m_{+}, m_{-}\right)$: $\operatorname{rank}\|G(\theta)\|=\operatorname{rank}\|G(0)\|, \operatorname{dim} \Sigma=\left.\operatorname{dim} \Sigma\right|_{\theta=0}$ with $\left.\Sigma\right|_{\theta=0}$ parameterized by $z^{i}(0)$. We characterize the property of a quantity $F$ to be bosonic or fermionic by a triplet of $Z_{2}$-gradings, $\vec{\varepsilon}=\left(\varepsilon_{P}, \varepsilon_{\bar{J}}, \varepsilon\right)$, so that the basic Grassmann parity $\varepsilon$, according to $[1,2,4]$, is given by the sum, $\varepsilon=\varepsilon_{J}+\varepsilon_{P}$, of $Z_{2}$-gradings $\varepsilon_{\bar{J}}, \varepsilon_{P}$, being the Grassmann parities of coordinates of the corresponding representation spaces of supergroups $\bar{J}, P$.

\section{CLASSICAL FORMULATION OF A DEGENERATE $\theta$-LOCAL SUPERFIELD MODEL}

In order to provide an equivalent description of a general LSM degenerate with respect to superfields $\left(\partial_{\theta} \mathcal{A}\right)^{I}(\theta)$ in oddLagrangian and odd-Hamiltonian formulations, we consider a procedure of the odd Hamiltonization of a Lagrangian LSM that preserves $\bar{J}$-covariance. Using these results, in the next section we will apply the BFV-BRST approach in order to construct from the odd Hamiltonian formulation of an LSM a complete Hamiltonian action $S_{\mathrm{H}}(\theta)$ and extend the BV-BFV dual description inherent in the $\theta$-local approach to the case of a degenerate LSM. We will also establish a relation with the quantum action of the BV method for the physical gauge model contained in an LSM.

\subsection{Odd-Lagrangian Formulation}

Let us recall that the Lagrangian formulation (based on the variational principle) of an $L_{g}$-stage reducible LSM of generalized classical superfields $\mathcal{A}^{I}(\theta), \vec{\varepsilon}\left(\mathcal{A}^{I}\right)=\left(\left(\varepsilon_{P}\right)_{I},\left(\varepsilon_{\bar{J}}\right)_{I}, \varepsilon_{I}\right) \equiv$ $\vec{\varepsilon}_{I}$, is defined by a Lagrangian action $S_{\mathrm{L}}, \Pi T \mathcal{M}_{\mathrm{CL}} \times\{\theta\} \rightarrow$ $\Lambda_{1}(\theta ; \mathbb{R})$, being a $C^{\infty}\left(\Pi T \mathcal{M}_{\mathrm{CL}}\right)$-function taking its values in a real Grassmann algebra $\Lambda_{1}(\theta ; \mathbb{R})$, and (independently) by a functional $Z_{\mathrm{L}}[\mathcal{A}]$, whose $\theta$-density is defined with accuracy up to an arbitrary $\vec{\varepsilon}$-bosonic function $f\left(\left(\mathcal{A}, \partial_{\theta} \mathcal{A}\right)(\theta), \theta\right) \in$ $\operatorname{ker}\left\{\partial_{\theta}\right\}$,

$$
Z_{\mathrm{L}}[\mathcal{A}]=\partial_{\theta} S_{\mathrm{L}}(\theta), \vec{\varepsilon}\left(Z_{\mathrm{L}}\right)=\vec{\varepsilon}(\theta)=(1,0,1), \vec{\varepsilon}\left(S_{\mathrm{L}}\right)=\overrightarrow{0}
$$

invariant with respect to general gauge transformations, $\delta_{g} \mathcal{A}^{I}(\theta)=\int d \theta_{0} \hat{\mathcal{R}}_{\mathscr{A}_{0}}^{I}\left(\theta ; \theta_{0}\right) \xi^{\mathcal{A}_{0}}\left(\theta_{0}\right), \xi^{\mathcal{A}_{0}} \in C^{\infty}(\mathcal{M}), \vec{\varepsilon}\left(\xi^{\mathcal{A}_{0}}\right)=\vec{\varepsilon}_{\mathcal{A}_{0}}, \mathcal{A}_{0}=$ $1, \ldots, M_{0}=M_{0+}+M_{0-}$ :

$$
\partial_{\theta} \frac{\overleftarrow{\delta} Z_{\mathrm{L}}[\mathcal{A}]}{\delta \mathcal{A}^{I}(\theta)} \hat{\mathcal{R}}_{\mathscr{A}_{0}}^{I}\left(\theta ; \theta_{0}\right)=0, \text { for rank }\left\|\overrightarrow{\mathcal{L}}_{J}(\theta)\left[\overleftarrow{\mathcal{L}}_{I}(\theta) S_{\mathrm{L}}(\theta)\right]\right\|_{\overleftarrow{L}_{K} S_{\mathrm{L}}=0}=\bar{N}-\bar{M}_{-1}
$$


with a superfield Euler-Lagrange derivative $\overleftarrow{L}_{I}(\theta)$ that determines LSM dynamics and (on the assumption of locality and $\bar{J}$ covariance) with functionally dependent generators $\hat{\mathcal{R}}_{\mathscr{A}_{0}}^{I}\left(\theta ; \theta_{0}\right)$

$$
\begin{gathered}
\frac{\overleftarrow{\delta} Z_{\mathrm{L}}[\mathcal{A}]}{\delta \mathcal{A}^{I}(\theta)}=\left[\frac{\partial}{\partial \mathcal{A}^{I}(\theta)}-(-1)^{\varepsilon_{I}} \overleftarrow{\partial}_{\theta} \frac{\overleftarrow{\partial}}{\partial\left(\overleftarrow{\partial}_{\theta} \mathcal{A}^{I}(\theta)\right)}\right] S_{\mathrm{L}}(\theta) \equiv \overleftarrow{\mathcal{L}}_{I}(\theta) S_{\mathrm{L}}(\theta)=0 \\
\hat{\mathfrak{R}}_{\mathfrak{A}_{0}}^{I}\left(\theta ; \theta_{0}\right)=\sum_{k \geq 0}\left(\left(\partial_{\theta}\right)^{k} \delta\left(\theta-\theta_{0}\right)\right) \hat{\mathcal{R}}_{k}^{I} \mathcal{A}_{0}\left(\left(\mathcal{A}, \partial_{\theta} \mathcal{A}\right)(\theta), \theta\right) \text { for rank }\left\|\sum_{k \geq 0} \hat{\mathcal{R}}_{k}^{I} \mathcal{A}_{0}(\theta)\left(\partial_{\theta}\right)^{k}\right\|_{\mathcal{L}_{K} S_{\mathrm{L}}=0}=\bar{M}_{-1}<\bar{M}_{0}
\end{gathered}
$$

The dependence of $\hat{\mathscr{R}}_{\mathscr{A}_{0}}^{I}\left(\theta ; \theta_{0}\right)$ implies an existence (on solutions of the odd LS (2.3)) of proper zero-eigenvalue eigenvectors, $\hat{z}_{\mathcal{A}_{1}}^{\mathcal{A}_{0}}\left(\mathcal{A}\left(\theta_{0}\right), \partial_{\theta_{0}} \mathcal{A}\left(\theta_{0}\right), \theta_{0} ; \theta_{1}\right)$, with a structure similar to $\hat{R}_{\mathscr{A}_{0}}^{I}\left(\theta ; \theta_{0}\right)$ in (2.4), which exhaust the zero-modes of the generators and are dependent in case rank $\left\|\sum_{k} \hat{z}_{k}^{\mathcal{A}_{0}}\left(\theta_{0}\right)\left(\partial_{\theta_{0}}\right)^{k}\right\|_{\Sigma}=\bar{M}_{0}-\bar{M}_{-1}<\bar{M}_{1}$. Thus, a general $L_{g}$-reducible LSM is defined by the reducibility relations, for $s=1, \ldots, L_{g}, \mathcal{A}_{s}=1, \ldots, M_{s}=M_{s+}+M_{s-}$,

$$
\begin{aligned}
& \int d \theta^{\prime} \hat{\mathcal{Z}}_{\mathcal{A}_{s-1}}^{\mathcal{A}_{s-2}}\left(\theta_{s-2} ; \theta^{\prime}\right) \hat{Z}_{\mathcal{A}_{s}}^{\mathcal{A}_{s-1}}\left(\theta^{\prime} ; \theta_{s}\right)=\int d \theta^{\prime} \vec{L}_{J}\left(\theta^{\prime}\right) L_{\mathcal{A}_{s}}^{\mathcal{A}_{s-2} J}\left(\left(\mathcal{A}, \partial_{\theta} \mathcal{A}\right)\left(\theta_{s-2}\right), \theta_{s-2}, \theta^{\prime} ; \theta_{s}\right),
\end{aligned}
$$

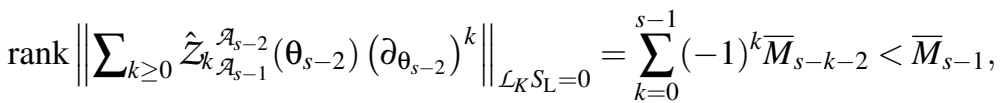

$$
\begin{aligned}
& \operatorname{rank}\left\|\sum_{k \geq 0} \hat{z}_{k_{\mathcal{L}_{L_{g}}}^{\mathcal{L}_{L_{g}-1}}\left(\theta_{L_{g}-1}\right)}\left(\partial_{\theta_{L_{g}-1}}\right)^{k}\right\|_{\mathcal{L}_{K} S_{\mathrm{L}}=0}=\sum_{k=0}^{L_{g}}(-1)^{k} \bar{M}_{L_{g}-k-1}=\bar{M}_{L_{g}}, \\
& \vec{\varepsilon}\left(\hat{z}_{\mathcal{A}_{s}}^{\mathcal{A}_{s-1}}\right)=\vec{\varepsilon}_{\mathcal{A}_{s-1}}+\vec{\varepsilon}_{\mathcal{A}_{s}}+(1,0,1), \hat{z}_{\mathcal{A}_{0}}^{\mathcal{A}_{1}}\left(\theta_{-1} ; \theta_{0}\right) \equiv \hat{\mathcal{R}}_{\mathfrak{A}_{0}}^{I}\left(\theta_{-1} ; \theta_{0}\right), \\
& L_{\mathcal{A}_{1}}^{\mathcal{A}_{1} J}\left(\theta_{-1}, \theta^{\prime} ; \theta_{1}\right) \equiv K_{\mathcal{A}_{1}}^{I J}\left(\theta_{-1}, \theta^{\prime} ; \theta_{1}\right)=-(-1)^{\left(\varepsilon_{I}+1\right)\left(\varepsilon_{J}+1\right)} K_{\mathcal{A}_{1}}^{J I}\left(\theta^{\prime}, \theta_{-1} ; \theta_{1}\right) .
\end{aligned}
$$

For $L_{g}=0$, the LSM is an irreducible general gauge theory.

Due to the $J$-scalar nature of $Z_{\mathrm{L}}[\mathcal{A}]$ it is only $S_{\mathrm{L}}(\theta)$, among the objects $S_{\mathrm{L}}(\theta)$ and $Z_{\mathrm{L}}[\mathcal{A}]$ invariant under the action of a $J$-superfield representation $T$ restricted to $\bar{J},\left.T\right|_{J}$, that transforms nontrivially with respect to the total representation $T$ under $\mathcal{A}^{I}(\theta) \rightarrow \mathcal{A}^{\prime I}(\theta)=\left(\left.T\right|_{J} \mathcal{A}\right)^{I}(\theta-\mu)$,

$$
\delta S_{\mathrm{L}}(\theta)=S_{\mathrm{L}}\left(\mathcal{A}^{\prime}(\theta), \partial_{\theta} \mathcal{A}^{\prime}(\theta), \theta\right)-S_{\mathrm{L}}(\theta)=-\mu\left[\vec{\partial} / \partial \theta+P_{0}(\theta)\left(\partial_{\theta} U\right)(\theta)\right] S_{\mathrm{L}}(\theta) .
$$

Eq. (2.6) is written $[1,2,4]$ in terms of the nilpotent operator $\left(\partial_{\theta} U\right)(\theta)=\partial_{\theta} \mathcal{A}^{I}(\theta) \vec{\partial} / \partial \mathcal{A}^{I}(\theta)=\left[\partial_{\theta}, U(\theta)\right]_{-}, U(\theta)=$ $P_{1} \mathcal{A}^{I}(\theta) \vec{\partial} / \partial \mathcal{A}^{I}(\theta)$, by means of projectors onto $C^{\infty}\left(\Pi T^{(*)} \mathcal{M}_{\mathrm{CL}}\right) \times\{\theta\}:\left\{P_{a}(\theta)=\delta_{a 0}\left(1-\theta \partial_{\theta}\right)+\delta_{a 1} \theta \partial_{\theta}, a=0,1\right\}$. The superfield Euler-Lagrange equations (2.3) are equivalent (in view of $\partial_{\theta}^{2} \mathcal{A}^{I}(\theta) \equiv 0$ ) to an odd LS characterized by $2 N$ formally second-order differential equations in $\theta$,

$$
\begin{aligned}
& \partial_{\theta}^{2} \mathcal{A}^{J}(\theta) \frac{\vec{\partial}}{\partial\left(\partial_{\theta} \mathcal{A}^{I}(\theta)\right)} \frac{\vec{\partial} S_{\mathrm{L}}(\theta)}{\partial\left(\partial_{\theta} \mathcal{A}^{J}(\theta)\right)} \equiv \partial_{\theta}^{2} \mathcal{A}^{J}(\theta)\left(S_{\mathrm{L}}^{\prime \prime}\right)_{I J}(\theta)=0, \\
& \Theta_{I}(\theta) \equiv(-1)^{\varepsilon_{I}}\left(S_{\mathrm{L}, I}(\theta)-\left[\frac{\vec{\partial}}{\partial \theta} \frac{\vec{\partial} S_{\mathrm{L}}(\theta)}{\partial\left(\partial_{\theta} \mathcal{A}^{I}(\theta)\right)}+\left(\partial_{\theta} U\right)(\theta) \frac{\vec{\partial} S_{\mathrm{L}}(\theta)}{\partial\left(\partial_{\theta} \mathcal{A}^{I}(\theta)\right)}\right]\right)=0,
\end{aligned}
$$

so that the subset of formal equations (2.7) does not affect LSM dynamics, in particular, identities (2.2) and relations (2.5); however, it determines, depending on the (non)degeneracy of the supermatrix $\left\|\left(S_{\mathrm{L}}^{\prime \prime}\right)_{I J}(\theta)\right\|$, a possibility of presenting LS (2.3) in the normal form. The Lagrangian constraints $(2.8), \Theta_{I}(\theta)=\Theta_{I}\left(\left(\mathcal{A}, \partial_{\theta} \mathcal{A}\right)(\theta), \theta\right)$ are functionally dependent as first-order equations in $\theta$, restricting the setting of the Cauchy problem for the LS, and thus determine the general gauge algebra of an LSM by Eqs. (2.2)-(2.5).
For an LSM which enables one to represent $S_{\mathrm{L}}(\theta)$ in the form of a natural system in usual classical mechanics, $S_{\mathrm{L}}(\theta)=T\left(\partial_{\theta} \mathcal{A}(\theta)\right)-S(\mathcal{A}(\theta), \theta)$, the functions $\Theta_{I}(\theta)$, $\Theta_{I}(\theta) \in C^{\infty}\left(\mathcal{M}_{\mathrm{CL}} \times\{\theta\}\right)$ are given by the relations

$$
\Theta_{I}(\theta)=-S, I(\mathcal{A}(\theta), \theta)(-1)^{\varepsilon_{I}}=0,
$$

being, for $\theta=0$, the extremals of the functional $S_{0}(A)=$ $S(\mathcal{A}(0), 0), S_{0}(A) \in C^{\infty}\left(M_{\mathrm{CL}}\right), M_{\mathrm{CL}}=\left.\mathcal{M}_{\mathrm{CL}}\right|_{\theta=0}$. Relations (2.2) and (2.5) assume the standard (in case $\theta=0$ ) form 
of the relations of a special gauge algebra with linearly dependent generators $\mathcal{R}_{0}^{I} \mathcal{A}_{0}(\mathcal{A}(\theta), \theta)$ of special gauge transformations, $\delta \mathcal{A}^{I}(\theta)=\mathcal{R}_{\mathcal{A}_{0}}^{I}(\mathcal{A}(\theta), \theta) \xi_{0}^{\mathcal{A}_{0}}(\theta)$, that leave invariant only $S(\theta)$, in contrast to $T(\theta)$,

$$
\begin{aligned}
& S, I(\mathcal{A}(\theta), \theta) \mathcal{R}_{\mathcal{A}_{0}}^{I}(\mathcal{A}(\theta), \theta)=0 \text {, for rank }\left\|S,_{I J}(\mathcal{A}(\theta), \theta)\right\|_{S_{, I}(\theta)=0}=\bar{N}-\bar{M}_{-1}, \\
& Z_{\mathcal{A}_{s-1}}^{\mathcal{A}_{s-2}}(\mathcal{A}(\theta), \theta) Z_{\mathcal{A}_{s}}^{\mathcal{A}_{s-1}}(\mathcal{A}(\theta), \theta)=S,{ }_{J}(\theta) L_{\mathcal{A}_{s}}^{\mathcal{A}_{s-2} J}(\mathcal{A}(\theta), \theta), \vec{\varepsilon}\left(Z_{\mathcal{A}_{s}}^{\mathcal{A}_{s-1}}\right)=\vec{\varepsilon}_{\mathcal{A}_{s-1}}+\vec{\varepsilon}_{\mathcal{A}_{s}}, \\
& \left(z_{\mathcal{A}_{0}}^{\mathcal{A}_{1}}, L_{\mathcal{A}_{1}}^{\mathcal{A}_{1} J}\right) \equiv\left(\mathcal{R}_{\mathcal{A}_{0}}^{I}, K_{\mathcal{A}_{1}}^{I J}\right), K_{\mathcal{A}_{1}}^{I J}=-(-1)^{\varepsilon_{I} \varepsilon_{J}} K_{\mathcal{A}_{1}}^{J I},
\end{aligned}
$$

in the rank conditions for the special zero-eigenvalue eigenvectors $Z_{\mathcal{A}_{s}}^{\mathcal{A}_{s-1}}(\theta)$ :

$$
\operatorname{rank}\left\|z_{\mathcal{A}_{s}}^{\mathcal{A}_{s-1}}\right\|_{S, I(\theta)=0}=\sum_{k=0}^{s}(-1)^{k} \bar{M}_{s-k-1}<\bar{M}_{s}, s=0, \ldots, L_{g}-1 .
$$

In case rank $\left\|z_{\mathcal{A}_{L_{g}}}^{\mathcal{A}_{L g}-1}\right\|_{S_{, I}(\theta)=0}=\sum_{k=0}^{L_{g}}(-1)^{k} \bar{M}_{L_{g}-k-1}=\bar{M}_{L_{g}}$, an LSM defined by relations (2.10), (2.11) is called a special gauge theory of $L_{g}$-stage reducibility.

The special gauge algebra of this LSM is $\theta$-locally embedded into the gauge algebra of a general gauge theory with the functional $Z_{\mathrm{L}}[\mathcal{A}]=\left(\partial_{\theta} T-S\right)(\theta)$, which leads to a relation between the eigenvectors,

$$
\hat{Z}_{\mathcal{A}_{s}}^{\mathcal{A}_{s-1}}\left(\mathcal{A}\left(\theta_{s-1}\right), \theta_{s-1} ; \theta_{s}\right)=\delta\left(\theta_{s}-\theta_{s-1}\right) Z_{\mathcal{A}_{s}}^{\mathcal{A}_{s-1}}\left(\mathcal{A}\left(\theta_{s-1}\right), \theta_{s-1}\right)
$$

and to a possible parametric dependence of the structure functions of a special gauge theory on $\partial_{\theta} \mathcal{A}^{I}(\theta)$. As noted in [1, 4], an extended (in comparison with $\left\{P_{a}(\theta)\right\}, a=0,1$ ) system of projectors onto $C^{\infty}\left(\Pi T \mathcal{M}_{\mathrm{CL}} \times\{\theta\}\right),\left\{P_{0}(\theta), \theta \partial / \partial \theta, U(\theta)\right\}$, selects in (2.10), (2.11) two kinds of gauge algebra: one is selected by means of the subsystem $\left\{P_{0}(\theta), U(\theta)\right\}$, with the corresponding structure equations and functions $\left[S, Z_{\mathcal{A}_{s}}^{\mathcal{A}_{s-1}}\right](\mathcal{A}(\theta))$ not depending explicitly on $\theta$, and another with the help of $P_{0}(\theta)$ with the standard (in case $\theta=0,\left(\varepsilon_{P}\right)_{I}=\left(\varepsilon_{P}\right)_{\mathcal{A}_{s}}=0$, $s=1, \ldots, L_{g}$ ) relations for the gauge algebra of a reducible model with quantities $\left[S_{0}, Z_{\mathrm{\alpha}_{s}}^{\alpha_{s-1}}\right](A)$ under the assumption of the completeness of reduced generators and eigenvectors $\left[\mathcal{R}_{\alpha_{0}}^{i}, Z_{\alpha_{s}}^{\alpha_{s-1}}\right](\mathcal{A}(\theta))$.

As shown in Refs. $[1,2,4]$, a characteristic feature of the $\theta$ local extension of a usual field theory to an LSM is the application of Noether's first theorem [19] to provide the invariance of the density $d \theta S_{\mathrm{L}}(\theta)$ under global $\theta$-translations as symmetry transformations of the superfields $\mathcal{A}^{I}(\theta)$ and coordinates $\left(z^{M}, \theta\right),\left(\mathcal{A}^{I}, z^{M}, \theta\right) \rightarrow\left(\mathcal{A}^{I}, z^{M}, \theta+\mu\right)$. It is easy to see that the function

$$
S_{\mathrm{E}}\left(\left(\mathcal{A}, \partial_{\theta} \mathcal{A}\right)(\theta), \theta\right) \equiv \frac{\overleftarrow{\partial} S_{\mathrm{L}}(\theta)}{\partial\left(\overleftarrow{\partial}_{\theta}^{r} \mathcal{A}^{I}(\theta)\right)} \overleftarrow{\partial}_{\theta} \mathcal{A}^{I}(\theta)-S_{\mathrm{L}}(\theta)
$$

is an LS integral of motion, namely, a quantity preserved by $\theta$-evolution, assuming the fulfillment of the generalized La- grangian master equation

$$
\frac{\vec{\partial}}{\partial \theta} S_{\mathrm{L}}(\theta)+\left.2\left(\partial_{\theta} U\right)(\theta) S_{\mathrm{L}}(\theta)\right|_{\mathcal{L}_{I} S_{\mathrm{L}}=0}=0 .
$$

Eq. (2.15) follows from the principle of dynamical symmetry in contrast to the standard (Hamiltonian-like) master equation in the minimal sector of the BV method [3] which is based on differential-algebraic reasons as a generating equation encoding the standard relations of the gauge algebra and the structure functions $\left[S_{0}, Z_{\alpha_{s}}^{\alpha_{s-1}}\right](A)$. The function $S_{\mathrm{E}}(\theta)$ may also be an LS integral in the case of an explicit dependence on $\theta$, unlike its analogue in a $t$-local field theory, the energy $E(t)$, in case $S_{\mathrm{L}}(\theta)$ admits the representation

$$
S_{\mathrm{L}}\left(\left(\mathcal{A}, \partial_{\theta} \mathcal{A}\right)(\theta), \theta\right)=
$$

$$
S_{\mathrm{L}}^{0}\left(\mathcal{A}, \partial_{\theta} \mathcal{A}\right)(\theta)-2 \theta\left(\partial_{\theta} U\right)(\theta) S_{\mathrm{L}}^{0}(\theta), \vec{\varepsilon}\left(S_{\mathrm{L}}^{0}\right)=\overrightarrow{0} .
$$

If $S_{\mathrm{L}}(\theta)$ does not depend on $\theta$ explicitly, $\frac{\partial}{\partial \theta} S_{\mathrm{L}}(\theta)=0$, Eq. (2.15) transforms into a Lagrangian master equation, $\left.\left(\partial_{\theta} U\right)(\theta) S_{\mathrm{L}}(\theta)\right|_{\mathcal{L}_{I} S_{\mathrm{L}=0}}=0$. As was announced in Ref. [2], a sufficient condition for the existence of a proper solution $\hat{S}_{L}(\theta)$ for the Lagrangian master equation is the presence of an independent special Lagrangian constraints for a certain division of the index $I$,

$$
\left(\partial_{\theta} \mathcal{A}^{A_{1}}(\theta), \vec{\partial} S_{\mathrm{L}}(\theta) / \partial \mathcal{A}^{A_{2}}(\theta)\right)_{\mathcal{L}_{I} S_{\mathrm{L}}=0}=0,\left(A_{1} \sqcup A_{2}\right)=I .
$$




\subsection{Odd Hamiltonization}

A possibility of presenting the odd LS (2.3) in the normal form (which, in a $t$-local field theory, provides a basis for the generalized canonical quantization of a given dynamical system) depends on the existence of an inverse for the supermatrix $\left\|\left(S_{\mathrm{L}}^{\prime \prime}\right)_{I J}(\theta)\right\|$ in (2.7).

\subsubsection{Nondegenerate Case}

In this case, the LSM is reformulated in the oddHamiltonian description $[1,2,4]$ on the odd phase space $\Pi T^{*} \mathcal{M}_{\mathrm{CL}}=\left\{\Gamma_{\mathrm{CL}}^{P}(\theta)=\left(\mathcal{A}^{I}, \mathcal{A}_{I}^{*}\right)(\theta)\right\}$ in terms of a Hamiltonian action being a $C^{\infty}\left(\Pi T^{*} \mathcal{M}_{\mathrm{CL}}\right)$-function, $S_{\mathrm{H}}$ : $\Pi T^{*} \mathcal{M}_{\mathrm{CL}} \times\{\theta\} \rightarrow \Lambda_{1}(\theta ; \mathbb{R})$, constructed from the Lagrangian formulation through a Legendre transformation of $S_{\mathrm{L}}(\theta)$ with respect to $\overleftarrow{\partial}_{\theta} \mathcal{A}^{I}(\theta)$

$$
S_{\mathrm{H}}\left(\Gamma_{\mathrm{CL}}(\theta), \theta\right)=\mathcal{A}_{I}^{*}(\theta) \overleftarrow{\partial}_{\theta} \mathcal{A}^{I}(\theta)-S_{\mathrm{L}}(\theta), \mathcal{A}_{I}^{*}(\theta)=\frac{\overleftarrow{\partial} S_{\mathrm{L}}(\theta)}{\partial\left(\overleftarrow{\partial}_{\theta} \mathcal{A}^{I}(\theta)\right)}, \vec{\varepsilon}\left(\mathcal{A}_{I}^{*}\right)=\vec{\varepsilon}_{I}+(1,0,1)
$$

The action $S_{\mathrm{H}}(\theta)$ coincides with $S_{\mathrm{E}}(\theta)$ in terms of the $\Pi T^{*} \mathcal{M}_{\mathrm{CL}}$-coordinates.

An odd HS equivalent to the LS follows from (2.3) due to transformations (2.18) and is implied by the condition of the existence of a critical superfield configuration for a fermionic functional $Z_{\mathrm{H}}\left[\Gamma_{\mathrm{CL}}\right]$ identical to $Z_{\mathrm{L}}[\mathcal{A}]$,

$$
\begin{aligned}
& Z_{\mathrm{H}}\left[\Gamma_{k}\right]=\int d \theta\left[V_{P}\left(\Gamma_{k}(\theta)\right) \overleftarrow{\partial}_{\theta} \Gamma_{k}^{P}(\theta)-S_{\mathrm{H}}\left(\Gamma_{k}(\theta), \theta\right)\right], \\
& \vec{\delta} Z_{\mathrm{H}} \\
& \frac{\delta \Gamma_{k}^{P}(\theta)}{}=\omega_{P Q}^{k}\left(\Gamma_{k}(\theta)\right)\left[\partial_{\theta} \Gamma_{\mathrm{k}}^{Q}(\theta)-\left(S_{\mathrm{H}}(\theta), \Gamma_{k}^{Q}(\theta)\right)_{\theta}\right]=0, k=\mathrm{CL} ;
\end{aligned}
$$

it is written in the square brackets in Eq. (2.20) with the help of a $\theta$-local antibracket and an antisymplectic potential, $V_{P}\left(\Gamma_{k}(\theta)\right)=$ $1 / 2\left(\Gamma^{Q} \omega_{Q P}^{k}\right)(\theta)$, defined with respect to an odd Poisson bivector $\omega_{k}^{P Q}(\theta), \omega_{k}^{P Q}(\theta) \equiv\left(\Gamma_{k}^{P}(\theta), \Gamma_{k}^{Q}(\theta)\right)_{\theta}$, and a flat antisymplectic metric $\omega_{P Q}^{k}(\theta), \omega_{k}^{P D}(\theta) \omega_{D Q}^{k}(\theta)=\delta^{P}{ }_{Q},\left[\omega_{k}^{P Q}, \omega_{P Q}^{k}\right](\theta)=\operatorname{antidiag}\left[\left(-\delta_{J}^{I}, \delta_{J}^{I}\right),\left(\delta_{J}^{I},-\delta_{J}^{I}\right)\right]$.

The equivalence of HS (2.20) and LS (2.7), (2.8) is provided by the fact that the Lagrangian constraints $\Theta_{I}(\theta)$ transformed into the Hamiltonian constraints $\Theta_{I}^{\mathrm{H}}(\theta)$ in terms of the $\Pi T^{*} \mathcal{M}_{\mathrm{CL}}$-coordinates coincide with half of the HS equations due to transformations (2.18) and their consequences:

$$
\Theta_{I}^{\mathrm{H}}\left(\Gamma_{k}(\theta), \theta\right)=\Theta_{I}\left(\mathcal{A}(\theta), \partial_{\theta} \mathcal{A}\left(\Gamma_{k}(\theta), \theta\right), \theta\right), \Theta_{I}^{\mathrm{H}}\left(\Gamma_{k}(\theta), \theta\right)=-\overleftarrow{\partial}_{\theta} \mathcal{A}_{I}^{*}(\theta)-S_{\mathrm{H}, I}(\theta)(-1)^{\varepsilon_{I}}
$$

The equivalence between the LS and HS is guaranteed by the corresponding [formal, in view of the degeneracy conditions (2.2)] setting of the Cauchy problem $(\theta=0, k=\mathrm{CL})$ for integral curves $\hat{\mathcal{A}}^{I}(\theta), \hat{\Gamma}_{k}^{P}(\theta)$, modulo the continuous part of $I$,

$$
\left(\hat{\mathcal{A}}^{I}, \vec{\partial}_{\theta} \hat{\mathcal{A}}^{I}\right)(0)=\left(\overline{\mathcal{A}}^{I}, \vec{\partial}_{\theta} \mathcal{A}^{I}\right), \hat{\Gamma}_{k}^{P}(0)=\left(\overline{\mathcal{A}}^{I}, \overline{\mathcal{A}}_{I}^{*}\right): \overline{\mathcal{A}}_{I}^{*}=P_{0}\left[\frac{\partial S_{\mathrm{L}}(\theta)}{\partial\left(\partial_{\theta} \mathcal{A}^{I}(\theta)\right)}\right]\left(\overline{\mathcal{A}}^{I}, \vec{\partial}_{\theta} \mathcal{A}^{I}\right) .
$$

The definition of a special gauge algebra (2.10), (2.11) remains the same in the Hamiltonian formulation, whereas the definition (2.2), (2.3) of a general $L_{g}$-stage reducible LSM is transformed by the rule

$$
\begin{aligned}
& \hat{Z}_{\mathrm{H}}{\stackrel{\mathcal{A}}{\mathcal{A}_{s}-1}}^{\mathcal{A}_{s}}\left(\Gamma_{k}\left(\theta_{s-1}\right), \theta_{s-1} ; \theta_{s}\right)=\hat{Z}_{\mathcal{A}_{s-1}}^{\mathcal{A}_{s-1}}\left(\mathcal{A}\left(\theta_{s-1}\right), \partial_{\theta_{s-1}} \mathcal{A}\left(\Gamma_{k}\left(\theta_{s-1}\right), \theta_{s-1}\right), \theta_{s-1} ; \theta_{s}\right), s=0, \ldots, L_{g}, \\
& \quad \frac{\delta Z_{\mathrm{L}}[\mathcal{A}]}{\delta \mathcal{A}^{I}(\theta)}=\frac{\delta Z_{\mathrm{H}}\left[\Gamma_{k}\right]}{\delta \mathcal{A}^{I}(\theta)}, \quad \int d \theta \frac{\overleftarrow{\delta} Z_{\mathrm{H}}\left[\Gamma_{k}\right]}{\delta \mathcal{A}^{I}(\theta)} \hat{\mathcal{R}}_{\mathcal{H}_{\mathcal{H}_{0}}}^{I}\left(\Gamma_{k}(\theta), \theta ; \theta_{0}\right)=0
\end{aligned}
$$

The fulfillment of the generalized Lagrangian master equation (2.15) for $S_{\mathrm{L}}(\theta)$ implies, in view of definition (2.14), transformations (2.18) and their consequence, $\frac{\partial}{\partial \theta}\left(S_{\mathrm{L}}+S_{\mathrm{H}}\right)(\theta)=0$, that the Hamiltonian action is an HS integral of motion (i.e., a quantity invariant under $\theta$-shifts along arbitrary solutions $\hat{\Gamma}_{k}^{P}(\theta)$ by $\left(\varepsilon_{P}, \varepsilon\right)$-odd $\mu$ ) due to a generalized Hamiltonian master equation,

$$
\mathbf{Q}_{\text {compl }}(\theta) S_{\mathrm{H}}(\theta) \equiv\left[\partial / \partial \theta-\left(S_{\mathrm{H}}(\theta), \quad\right)_{\theta}\right] S_{\mathrm{H}}(\theta)=0 \Longleftrightarrow\left(\left.\delta_{\mu} S_{\mathrm{H}}(\theta)\right|_{\hat{\Gamma}_{k}(\theta)}=\mu \mathbf{Q}_{\mathrm{compl}}(\theta) S_{\mathrm{H}}(\theta)=0\right) .
$$

The equation $\mathbf{Q}_{\text {compl }}(\theta) S_{\mathrm{H}}(\theta)=0$, written in terms of an odd operator $\mathbf{Q}_{\text {compl }}(\theta)$, holds true also in the case of an explicit dependence of $S_{\mathrm{H}}(\theta)$ on $\theta$, according to (2.16),

$$
S_{\mathrm{H}}\left(\Gamma_{k}(\theta), \theta\right)=S_{\mathrm{H}}^{0}\left(\Gamma_{k}(\theta)\right)+\theta\left(S_{\mathrm{H}}^{0}\left(\Gamma_{k}(\theta)\right), S_{\mathrm{H}}^{0}\left(\Gamma_{k}(\theta)\right)\right)_{\theta},
$$


where $S_{\mathrm{H}}^{0}(\theta)$ is a Legendre transform of $S_{\mathrm{L}}^{0}(\theta)$, and $\left(\partial_{\theta} U\right)(\theta) S_{\mathrm{L}}(\theta)=\frac{1}{2}\left(S_{\mathrm{H}}(\theta), S_{\mathrm{H}}(\theta)\right)_{\theta}$. If $S_{\mathrm{H}}(\theta)$ does not depend on $\theta$ explicitly, $(\partial / \partial \theta) S_{\mathrm{H}}(\theta)=0$; then Eq. (2.25) transforms into the Hamiltonian master equation $\left(S_{\mathrm{H}}(\theta), S_{\mathrm{H}}(\theta)\right)_{\theta}=0$. This imposes the condition [3] for $S_{\mathrm{H}}(\theta)$ to be proper, which has no counterpart in a $t$-local field theory. Sufficient conditions of the solvability of the latter equation is the presence of irreducible special Hamiltonian constraints $\varphi_{a}\left(\Gamma_{k}(\theta)\right)$ [2], equivalent to the constraints (2.17), being of first-class with respect to the antibracket:

$$
\begin{aligned}
& \varphi_{a}(\theta)=\left(\frac{\partial S_{\mathrm{H}}(\theta)}{\partial \mathcal{A}_{A_{1}}^{*}(\theta)}, \frac{\vec{\partial} S_{\mathrm{H}}(\theta)}{\partial \mathcal{A}^{A_{2}(\theta)}}\right)=0,\left(\varphi_{a}(\theta), \varphi_{b}(\theta)\right)_{\theta}=C_{a b}^{c}\left(\Gamma_{k}(\theta)\right) \varphi_{c}(\theta), \\
& \operatorname{rank}\left\|\frac{\overleftarrow{\partial} \varphi_{a}(\theta)}{\partial \Gamma_{k}^{P}(\theta)}\right\|_{\varphi_{a}(\theta)=0, \hat{\Gamma}_{k}}=\bar{M}^{\mathrm{sp}} \leq \bar{N}+\left(N_{-}, N_{+}\right), M^{\mathrm{sp}}=\frac{1}{2} N .
\end{aligned}
$$

As a consequence, the $\theta$-superfield integrability of HS (2.20) is guaranteed by the properties of the antibracket, including the Jacobi identity

$$
\left(\overleftarrow{\partial}_{\theta}\right)^{2} \Gamma_{k}^{P}(\theta)=\frac{1}{2}\left(\Gamma_{k}^{P}(\theta),\left(S_{\mathrm{H}}\left(\Gamma_{k}(\theta)\right), S_{\mathrm{H}}\left(\Gamma_{k}(\theta)\right)\right)_{\theta}\right)_{\theta}=0
$$

and the $\theta$-translation formula written in terms of a nilpotent BRST-like generator $\breve{s}^{l}(\theta)$ of $\theta$-shifts along an $\left(\varepsilon_{P}, \varepsilon\right)$-odd vector field $\mathbf{Q}(\theta) \equiv \operatorname{ad} S_{\mathrm{H}}(\theta)=\left(S_{\mathrm{H}}(\theta), \cdot\right)_{\theta}$, acting on $C^{\infty}\left(\Pi T^{*} \mathcal{M}_{\mathrm{CL}} \times\{\theta\}\right)$, namely,

$$
\left.\delta_{\mu} \mathcal{F}(\theta)\right|_{\hat{\Gamma}_{k}(\theta)}=\mu \mathbf{Q}_{\text {compl }}(\theta) \mathcal{F}(\theta) \equiv \mu \check{s}^{l}(\theta) \mathcal{F}(\theta) .
$$

Following Refs. [1,2,4] and depending on the realization of additional properties of a gauge theory, we assume the fulfillment of the equation

$$
\begin{aligned}
\Delta^{k}(\theta) S_{\mathrm{H}}(\theta)=0, \Delta^{k}(\theta) & \equiv \frac{(-1)^{\varepsilon\left(\Gamma^{P}\right)}}{2} \rho^{-1} \frac{\vec{\partial}}{\partial \Gamma_{k}^{P}(\theta)}\left(\rho \omega_{k}^{P Q}\right)\left(\Gamma_{k}(\theta)\right) \frac{\vec{\partial}}{\partial \Gamma_{k}^{Q}(\theta)} \\
& =\frac{(-1)^{\varepsilon\left(\Gamma^{Q}\right)}}{2} \rho^{-1} \omega_{Q P}^{k}(\theta)\left(\Gamma_{k}^{P}(\theta), \rho\left(\Gamma_{k}^{Q}(\theta), \cdot\right)_{\theta}\right)_{\theta},
\end{aligned}
$$

for a trivial (in the case of a flat odd phase-space) choice of the density function $\rho(\Gamma(\theta)), \rho=1$, which is equivalent to a vanishing antisymplectic divergence of $\mathbf{Q}(\theta),\left(\vec{\partial} / \partial \Gamma_{k}^{P}(\theta)\right) \mathbf{Q}(\theta)=2 \Delta^{k}(\theta) S_{\mathrm{H}}(\theta)=0$.

\subsubsection{Degenerate Case}

The degeneracy of $\left\|\left(S_{\mathrm{L}}^{\prime \prime}\right)_{I J}(\theta)\right\|$ implies the impossibility of applying the above procedure of odd Hamiltonization and requires the use of an odd counterpart of Dirac's algorithm in order to reduce the odd LS (2.3) to a normal form (with only $\bar{J}$-covariance preservation). Let the degree of degeneracy of $\left\|\left(S_{\mathrm{L}}^{\prime \prime}\right)_{I J}(\theta)\right\|$ be given by the relation

$$
\operatorname{rank}\left\|\left(S_{\mathrm{L}}^{\prime \prime}\right)_{I J}(\theta)\right\|_{\overleftarrow{L}_{K} S_{\mathrm{L}}=0}=\overline{\Pi N}-\bar{R}_{-1}, \overline{\Pi N}=\left(N_{-}, N_{+}\right)
$$

valid almost everywhere in $\Pi T_{\mathrm{CL}}$. It means the impossibility to express each $\vec{\partial}_{\theta} \mathcal{A}^{I}(\theta)$ as a function of $\Gamma_{\mathrm{k}}^{P}(\theta)$ using equations $\mathcal{A}_{I}^{*}(\theta)=\left(\partial S_{\mathrm{L}}(\theta)\right) / \partial\left(\overleftarrow{\partial}_{\theta} \mathcal{A}^{I}(\theta)\right)$ in (2.18), which is equivalent to a functional dependence among the antifields $\mathcal{A}_{I}^{*}(\theta)$ in the form of primary antisymplectic constraints,

$$
\Phi_{A_{0}}^{(1)}\left(\Gamma_{\mathrm{k}}^{P}(\theta), \theta\right)=0, A_{0}=1, \ldots, R_{0}^{(0)}, \vec{\varepsilon}\left(\Phi_{A_{0}}^{(1)}\right)=\vec{\varepsilon}_{A_{0}}, \operatorname{rank}\left\|\left(\Phi_{A_{0}}^{(1)}, \Gamma_{\mathrm{k}}^{P}(\theta)\right)_{\theta} \omega_{P Q}^{k}\right\|_{\Phi_{A_{0}}^{(1)}=0}=\bar{R}_{-1} \leq \bar{R}_{0}^{(0)} .
$$

In view of the preservation of $\bar{J}$-covariance, the constraints $\Phi_{A_{0}}^{(1)}(\theta)$ may be dependent for $\bar{R}_{-1}<\bar{R}_{0}^{(0)}$, and assuming the existence of an analog of the regularity conditions [16], these constraints define a $\left(2 \bar{N}-\bar{R}_{-1}\right)$-dimensional submanifold smoothly embedded in $\Pi T_{\mathrm{CL}}^{*}$. The above regularity conditions imply the existence of an open covering of the constraints surface, $\Phi_{A_{0}}^{(1)}(\theta)=$ 0 , by open regions on each of which there exists a local separation of constraints:

$$
\Phi_{A_{0}}^{(1)}(\theta)=\left(\Phi_{A_{0}^{\prime}}^{(1)}, \Phi_{\alpha_{0}^{\prime}}^{(1)}\right)(\theta): \operatorname{rank}\left\|\left(\Phi_{A_{0}^{\prime}}^{(1)}, \Gamma_{k}^{P}(\theta)\right)_{\theta} \omega_{P Q}^{k}\right\|_{\Phi_{A_{0}}^{(1)}=0}=\bar{R}_{-1}, \Phi_{\alpha_{0}^{\prime}}^{(1)}(\theta)=\Phi_{A_{0}^{\prime}}^{(1)}(\theta) C_{\alpha_{0}^{\prime}}^{A_{0}^{\prime}}\left(\Gamma_{k}(\theta), \theta\right),
$$

where $A_{0}=\left(A_{0}^{\prime}, \alpha_{0}^{\prime}\right)=\left(1, \ldots, R_{-1}, 1, \ldots, R_{0}^{(0)}-R_{-1}\right)$.

For an LSM with primary constraints, there hold the following statements: 
Theorem 1 For any $C^{\infty}\left(\Pi T^{*} \mathcal{M}_{\mathrm{CL}}\right)$-function $\mathcal{F}\left(\Gamma_{\mathrm{CL}}(\theta), \theta\right)$ vanishing on the constraint surface, $\left.\mathcal{F}(\theta)\right|_{\Phi_{A_{0}}^{(1)}=0}=0$, there exist functions $f^{A_{0}}\left(\Gamma_{\mathrm{CL}}(\theta), \theta\right) \in C^{\infty}\left(\Pi T^{*} \mathcal{M}_{\mathrm{CL}}\right)$ such that $\mathcal{F}(\theta)$ is a linear combination of constraints: $\mathcal{F}(\theta)=\Phi_{A_{0}}^{(1)}(\theta) f^{A_{0}}(\theta)$.

Theorem 2 Due to the solution of the equation $\Lambda_{P}\left(\Gamma_{\mathrm{CL}}(\theta), \theta\right) \delta \Gamma_{\mathrm{CL}}^{P}(\theta)=0$ for arbitrary variations $\delta \Gamma_{\mathrm{CL}}^{P}(\theta)$, the functions

$$
\Lambda_{P}\left(\Gamma_{k}(\theta), \theta\right)=U^{A_{0}}\left(\Gamma_{k}(\theta), \theta\right)\left(\Phi_{A_{0}}^{(1)}(\theta), \Gamma_{k}^{Q}(\theta)\right)_{\theta} \omega_{Q P}^{k}(\theta), k=\mathrm{CL}
$$

are tangent to the constraints surface.

A sketch of a proof: the validity of Theorem 1 follows from the partition of unity on $\Pi T^{*} \mathcal{M}_{\mathrm{CL}}$ and from the fact that from the local validity of representation (2.33) follows the existence of an invertible change of variables, $\Gamma_{k}^{P}(\theta) \rightarrow \Gamma_{k}^{\prime P}(\theta)=$ $\left(\Phi_{A_{0}^{\prime}}^{(1)}, X^{M_{0}}\right)\left(\Gamma_{k}(\theta)\right), M_{0}=1, \ldots, 2 N-R_{-1}$, so that $\Phi_{A_{0}}^{(1)}\left(0, X^{M_{0}}\right)=0$. Therefore, in a regular coordinate system $\Gamma_{k}^{\prime}$ one has

$$
\begin{aligned}
\mathcal{F}\left(\Gamma_{k}^{\prime}(\theta)\right) & =\int_{0}^{1} \partial_{t} \mathcal{F}\left(t \Phi_{A_{0}^{\prime}}^{(1)}, X\right)(\theta) d t=\Phi_{A_{0}^{\prime}}^{(1)}(\theta) \int_{0}^{1} \frac{\vec{\partial}}{\partial\left(t \Phi_{A_{0}^{\prime}}^{(1)}(\theta)\right)} \mathcal{F}\left(t \Phi_{A_{0}^{\prime}}^{(1)}, X\right)(\theta) d t= \\
& =\Phi_{A_{0}^{\prime}}^{(1)}(\theta) f^{A_{0}^{\prime}}\left(\Gamma^{\prime}\left(\Gamma_{k}(\theta)\right), \theta\right) \Longrightarrow \mathcal{F}(\theta)=\left(\Phi_{A_{0}}^{(1)} f^{A_{0}}\right)(\theta) \text { with } f^{\alpha_{0}^{\prime}}(\theta)=0 .
\end{aligned}
$$

The validity of Theorem 2 follows from the fact that the variations $\delta \Gamma_{k}^{P}(\theta)$ tangent to the surface $\Phi_{A_{0}}^{(1)}(\theta)=0$ at a certain point form a $\left(2 \bar{N}-\bar{R}_{-1}\right)$-dimensional superspace so that there exist $R_{-1}$ independent solutions of $\left(\Lambda_{P} \delta \Gamma_{\mathrm{k}}^{P}\right)(\theta)=0$. By virtue of the regularity conditions (2.33), the above solutions may be chosen as a linear combination (2.34) of the antisymplectic gradients, $\left[\left(\Phi_{A_{0}^{\prime}}^{(1)}, \Gamma_{k}^{P}(\theta)\right)_{\theta} \omega_{P Q}^{k}\right](\theta)$, with functions $U^{\alpha_{0}^{\prime}}(\theta)$ unambiguously defined for $R_{0}^{(0)}>R_{-1}$.

The Hamiltonian action $S_{\mathrm{H}}\left(\Gamma_{k}(\theta), \theta\right)$ in $(2.18)$ is well-defined as a Legendre transformation of $S_{\mathrm{L}}(\theta)$ only on the surface $\Phi_{A_{0}}^{(1)}(\theta)=0$. The definition of the Hamiltonian action by the rule (2.18) on the entire $\Pi T^{*} \mathcal{M}_{k}$ is possible due to an extension of $S_{\mathrm{H}}(\theta)$ by the addition of linear combination of constraints:

$$
S_{\mathrm{H}}^{(1)}\left(\left(\Gamma_{k}, \Lambda\right)(\theta), \theta\right)=S_{\mathrm{H}}(\theta)+\Lambda^{A_{0}}(\theta) \Phi_{A_{0}}^{(1)}(\theta), \vec{\varepsilon}\left(\Lambda^{A_{0}}\right)=\vec{\varepsilon}_{A_{0}} .
$$

Actually, Eqs. (2.18) implies the following equations under a variation on the constraints surface:

$$
\left(S_{\mathrm{H}, I}(\theta)+S_{\mathrm{L}, I}(\theta)\right) \delta \mathcal{A}^{I}(\theta)+\delta \mathcal{A}_{I}^{*}(\theta)\left(\frac{\vec{\partial} S_{\mathrm{H}}}{\partial \mathcal{A}_{I}^{*}(\theta)}-\overleftarrow{\partial}_{\theta} \mathcal{A}^{I}(\theta)\right)=0
$$

which, in accordance with Theorem 2, admit the solution

$$
\left[\overleftarrow{\partial}_{\theta} \mathcal{A}^{I} ;-S_{\mathrm{L}, I}\right](\theta)=\left[\operatorname{ad} S_{\mathrm{H}}\left(\mathcal{A}^{I}\right)+\Lambda^{A_{0}} \operatorname{ad} \Phi_{A_{0}}^{(1)}\left(\mathcal{A}^{I}\right) ; S_{\mathrm{H}, I}+\Lambda^{A_{0}} \operatorname{ad} \Phi_{A_{0}}^{(1)}\left(\mathcal{A}_{I}^{*}\right)\right](\theta)
$$

In the case of independent constraints, the unknown functions $\Lambda^{A_{0}}(\theta)$ can be uniquely obtained from the first equations for $\overleftarrow{\partial}_{\theta} \mathcal{A}^{I}$ in (2.36) as functions on $\Pi T \mathcal{M}_{C L}, \Lambda^{A_{0}}=\Lambda^{A_{0}}\left(\mathcal{A}, \partial_{\theta} \mathcal{A}\right)$ for $\mathcal{A}_{I}^{*}=\mathcal{A}_{I}^{*}\left(\mathcal{A}, \partial_{\theta} \mathcal{A}\right)$ in contrast to dependent $\Phi_{A_{0}}^{(1)}(\theta)$

The invertible Legendre transformation, corresponding to Eqs. (2.36), of $S_{\mathrm{L}}(\theta)$ from $\Pi T \mathcal{M}_{C L}$ to the surface $\Phi_{A_{0}}^{(1)}(\theta)=0$ of THE bundle $\mathcal{N}_{C L}=\Pi T^{*} \mathcal{M}_{C L} \oplus \mathcal{M}_{\Lambda}=\left\{\left(\Gamma_{k}^{P}, \Lambda^{A_{0}}\right)\right\}$ and its inverse are given by the relations

$$
\begin{aligned}
& \left(\mathcal{A}^{I}, \mathcal{A}_{I}^{*}, \Lambda^{A_{0}}\right)(\theta)=\left(\mathcal{A}^{I}, \frac{\overleftarrow{\partial} S_{\mathrm{L}}(\theta)}{\partial\left(\overleftarrow{\partial}_{\theta} \mathcal{A}^{I}(\theta)\right)}, \Lambda^{A_{0}}\left(\mathcal{A}, \partial_{\theta} \mathcal{A}\right)\right)(\theta) \\
& \left(\mathcal{A}^{I}, \overleftarrow{\partial}_{\theta} \mathcal{A}^{I}, \Phi_{A_{0}}^{(1)}\right)(\theta)=\left(\mathcal{A}^{I}, \operatorname{ad} S_{\mathrm{H}}^{(1)}\left(\mathcal{A}^{I}\right), 0\right)(\theta)
\end{aligned}
$$

We assume that the local consideration of the Legendre transformation made in Eqs. (2.36)-(2.38) holds globally.

The corresponding odd HS equivalent to LS (2.3) is implied by relations (2.18), (2.35)-(2.38) following from the variational principle for a fermionic functional $Z_{\mathrm{H}}^{(1)}\left[\Gamma_{k}, \Lambda\right]$ extended, as compared with $Z_{\mathrm{H}}\left[\Gamma_{k}\right]$ in (2.19), by means of linear combinations of the constraints $\Phi_{A_{0}}^{(1)}(\theta)$ with superfields $\Lambda^{A_{0}}(\theta)$ as Lagrangian multipliers,

$$
\begin{aligned}
& Z_{\mathrm{H}}^{(1)}\left[\Gamma_{k}, \Lambda\right]=\int d \theta\left[V_{P}^{k}\left(\Gamma_{k}(\theta)\right) \overleftarrow{\partial}_{\theta} \mathcal{A}^{I}(\theta)-S_{\mathrm{H}}^{(1)}\left(\left(\Gamma_{k}, \Lambda\right)(\theta), \theta\right)\right] \\
& \left(\frac{\vec{\delta} Z_{\mathrm{H}}^{(1)}}{\delta \Gamma_{k}^{P}(\theta)}, \frac{\vec{\delta} Z_{\mathrm{H}}^{(1)}}{\delta \Lambda^{A_{0}}(\theta)}\right)=\left(\omega_{P Q}^{k}\left(\Gamma_{k}(\theta)\right)\left[\partial_{\theta} \Gamma_{\mathrm{k}}^{Q}(\theta)-\left(S_{\mathrm{H}}^{(1)}(\theta), \Gamma_{k}^{Q}(\theta)\right)_{\theta}\right],-\Phi_{A_{0}}^{(1)}(\theta)\right)=0 .
\end{aligned}
$$


A relation between the Cauchy problem (or the boundary problem) for an LS and HS should be specified in view of $\Phi_{A_{0}}^{(1)}(\theta)$, whereas the restriction of the action $\left.S_{\mathrm{H}}^{(1)}(\theta)\right|_{\Phi_{A_{0}}^{(1)}=0}$ remains an expression of the function $S_{\mathrm{E}}(\theta)$ in terms of $\Pi T^{*} \mathcal{M}_{\mathrm{CL}}$-coordinates.

By the Legendre transformation, the consequence (2.37) is a change of $S_{\mathrm{H}, I}(\theta)$ by $S_{\mathrm{H}}^{(1)}{ }_{I}(\theta)$ in expression (2.21) for the Hamiltonian constraints $\Theta_{I}^{H}\left(\left(\Gamma_{k}, \Lambda\right)(\theta), \theta\right)=\Theta_{I}^{(1) H}(\theta)$, preserving the equivalence of a generalized HS that consists of the constraints $\Theta_{I}^{(1) H}(\theta)$ and HS (2.40) itself. In this case, the definition of the gauge algebra (2.23) in case of a nondegenerate supermatrix $\left\|\left(S_{\mathrm{L}}^{\prime \prime}\right)_{I J}(\theta)\right\|$ is not affected by the change $Z_{\mathrm{H}}\left[\Gamma_{k}\right] \rightarrow Z_{\mathrm{H}}^{(1)}\left[\Gamma_{k}, \Lambda\right]$ by virtue of the identities $\delta Z_{\mathrm{H}} / \delta \mathcal{A}^{I}(\theta)=$ $\delta Z_{\mathrm{H}}^{(1)} / \delta \mathcal{A}^{I}(\theta)$ implied by Eqs. (2.37).

Locally, having assumed that condition (2.31) is fulfilled in a vicinity of the configurations $\left(\mathcal{A}_{0}^{I}, \partial_{\theta} \mathcal{A}_{0}^{I}\right)=(0,0)$ the structure of the primary constraints $\Phi_{A_{0}}^{(1)}$ can be specified. Now, as the regularity conditions (2.33), one can assume, while neglecting $\bar{J}$-covariance, that there exists a separation of the superfields $\left(\mathcal{A}^{I}, \partial_{\theta} \mathcal{A}^{I}, \mathcal{A}_{I}^{*}\right)$ compatible with (2.31),

$$
\left(\mathcal{A}^{I}, \partial_{\theta} \mathcal{A}^{I}, \mathcal{A}_{I}^{*}\right)=\left(\left(\mathcal{A}^{I_{0}}, \mathcal{A}^{A_{0}^{\prime}}\right),\left(\partial_{\theta} \mathcal{A}^{I_{0}}, \partial_{\theta} \mathcal{A}^{A_{0}^{\prime}}\right),\left(\mathcal{A}_{I_{0}}^{*}, \mathcal{A}_{A_{0}^{\prime}}^{*}\right)\right): \operatorname{rank}\left\|\left(S_{\mathrm{L}}^{\prime \prime}\right)_{I_{0} J_{0}}(\theta)\right\|_{\overleftarrow{L}_{K} S_{\mathrm{L}}=0}=\overline{\Pi N}-\bar{R}-1
$$

In this case, the action $S_{\mathrm{H}}\left(\left(\Gamma_{k}\right)(\theta), \theta\right) \equiv S_{\mathrm{H}}\left(\left(\mathcal{A}, \mathcal{A}_{I_{0}}^{*}\right)(\theta), \theta\right)$ depends neither on the antifields $\mathcal{A}_{A_{0}^{\prime}}$ nor on the primary nonexpressible superfields $\partial_{\theta} \mathcal{A}^{A_{0}^{\prime}}$ due to the direct Legendre transformation (2.37), in which, instead of $\Lambda^{A_{0}}$, one writes $\partial_{\theta} \mathcal{A}^{A_{0}^{\prime}}$, and due to the fact that the action $S_{\mathrm{L}}(\theta)$ is at most homogeneous of degree 1 with respect to $\partial_{\theta} \mathcal{A}^{A_{0}^{\prime}}$. The action $S_{\mathrm{H}}^{(1)}\left(\left(\Gamma_{k}, \Lambda\right)(\theta), \theta\right)$ has the form

$$
S_{\mathrm{H}}^{(1)}(\theta)=\left(S_{\mathrm{H}}+\Lambda^{A_{0}^{\prime}} \Phi_{A_{0}^{\prime}}^{(1)}\right)(\theta), \Phi_{A_{0}^{\prime}}^{(1)}(\theta)=\mathcal{A}_{A_{0}^{\prime}}^{*}(\theta)-\frac{\overleftarrow{\partial} S_{\mathrm{L}}(\theta)}{\partial\left(\overleftarrow{\partial}_{\theta} \mathcal{A}^{A_{0}^{\prime}}(\theta)\right)} \mid \partial_{\theta} \mathcal{A}_{0}^{I_{0}=\partial_{\theta} \mathcal{A}_{0}{ }^{\prime}\left(\mathcal{A}, \mathcal{A}_{I_{0}}^{*}, \partial_{\theta} \mathcal{A}^{A_{0}^{\prime}}\right)}
$$

where the odd velocities $\partial_{\theta} \mathcal{A}^{I_{0}}(\theta)$ are resolved from the relations $\mathcal{A}_{I_{0}}^{*}(\theta)=\frac{\overleftarrow{\partial} S_{\mathrm{L}}(\theta)}{\partial\left(\overleftarrow{\partial}_{\theta} \mathcal{A}_{0}(\theta)\right)}$. In turn, Eqs. (2.38) imply the coincidence of the Lagrangian multipliers $\Lambda^{A_{0}^{\prime}}$ and $\partial_{\theta} \mathcal{A}^{A_{0}^{\prime}}$.

Having obtained an equivalent description of the LSM in terms of the Hamiltonian action $S_{\mathrm{H}}^{(1)}(\theta)$, and following the $\theta$-local analog of Dirac's algorithm, we need to check the compatibility of the odd HS in (2.40), being the preservation of the constraints by the $\theta$-evolution generated by the vector field $\mathbf{Q}_{\text {compl }}^{(1)}(\theta), \mathbf{Q}_{\text {compl }}^{(1)}(\theta) \equiv\left[\partial / \partial \theta-\operatorname{ad} S_{\mathrm{H}}^{(1)}(\theta)\right]=\mathbf{Q}_{\mathrm{compl}}(\theta)-\Lambda^{A_{0}} \operatorname{ad} \Phi_{A_{0}}^{(1)}(\theta)$,

$$
\partial_{\theta} \Phi_{A_{0}}^{(1)}(\theta)=\mathbf{Q}_{\mathrm{compl}}(\theta)\left(\Phi_{A_{0}}^{(1)}(\theta)\right)-\Lambda^{B_{0}}\left(\Phi_{B_{0}}^{(1)}(\theta), \Phi_{A_{0}}^{(1)}(\theta)\right)_{\theta}=0
$$

Eqs. (2.43) may contain a subsystem with $R_{0}^{\prime(1)}$ equations independent from $\Lambda^{A_{0}}$ with residual $\left(R_{0}^{(0)}-R_{0}^{\prime(1)}\right)$ equations permitting to find a part of $\Lambda^{A_{0}}$ as functions of $\Gamma_{k}^{P}(\theta)$ if $\left.\left(\Phi_{A_{0}}^{(1)}(\theta), \Phi_{B_{0}}^{(1)}(\theta)\right)_{\theta}\right|_{\Phi^{(1)}=0} \neq 0$. In the former case, if a subsystem of $R_{0}^{(1)} \leq R_{0}^{(1)}$ equations does not depend on $\Phi_{A_{0}}^{(1)}(\theta)$, i.e., if there exists a set $\left\{\Phi_{A_{1}}^{(1)}(\theta)\right\}$ of $\left\{\Phi_{A_{0}}^{(1)}(\theta)\right\}$ such that

$$
\left(\mathbf{Q}_{\mathrm{compl}}^{(1)} \Phi_{A_{1}}^{(1)}\right)(\theta)=\left(\mathbf{Q}_{\mathrm{compl}} \Phi_{A_{1}}^{(1)}\right)(\theta)=U_{A_{1}}^{B_{0}}\left(\Gamma_{k}(\theta), \theta\right) \Phi_{B_{0}}^{(1)}(\theta), A_{1}=1, \ldots, R_{0}^{(1)} \text { for } U_{A_{1}}^{B_{0}}(\theta)=0
$$

The above expression defines dependent (in general) secondary antisymplectic constraints $\Phi_{A_{1}}^{(2)}(\theta) \in C^{\infty}\left(\Pi T^{*} \mathcal{M}_{k}\right)$,

$$
\Phi_{A_{1}}^{(2)}(\theta)=\left(\mathbf{Q}_{\mathrm{compl}} \Phi_{A_{1}}^{(1)}\right)(\theta)=0
$$

The constraints $\Phi_{A_{1}}^{(2)}(\theta)$, in contrast to $\Phi_{A_{0}}^{(1)}(\theta)$, are not valid identically in the entire $\Pi T^{*} \mathcal{M}_{k}$, but only on solutions of the odd HS (2.40).

The consistency conditions for the secondary constraints $\Phi_{A_{1}}^{(2)}(\theta)$ of the form $(2.43),\left(\mathbf{Q}_{\text {compl }}^{(1)} \Phi_{A_{1}}^{(2)}\right)(\theta)=0$, lead to new (generally dependent) secondary antisymplectic constraints, $\Phi_{A_{2}}^{(3)}(\theta)=\left(\mathbf{Q}_{\text {compl }} \Phi_{A_{2}}^{(2)}\right)(\theta)=0, A_{2}=1, \ldots, R_{0}^{(2)}, R_{0}^{(2)} \leq R_{0}^{(2)}=$ $R_{0}^{\prime(1)}-R_{0}^{(1)}$ among $R_{0}^{\prime(2)}$ equations not containing $\Lambda^{A_{0}}$ in the relations for the subset $\left\{\Phi_{A_{2}}^{(2)}\right\} \subseteq\left\{\Phi_{A_{2}}^{(1)}\right\}$,

$$
\left(\mathbf{Q}_{\mathrm{compl}}^{(1)} \Phi_{A_{2}}^{(2)}\right)(\theta)=\left(\mathbf{Q}_{\mathrm{compl}} \Phi_{A_{2}}^{(2)}\right)(\theta)=\left(U_{A_{2}}^{B_{0}} \Phi_{B_{0}}^{(1)}+U_{A_{2}}^{B_{1}} \Phi_{B_{1}}^{(2)}\right)(\theta), \text { for } U_{A_{2}}^{B_{0}}(\theta)=U_{A_{2}}^{B_{1}}(\theta)=0
$$


The remaining part of $\left(R_{0}^{(1)}-R_{0}^{(1)}-R_{0}^{(2)}\right)$ equations in $\left(\mathbf{Q}_{\text {compl }}^{(1)} \Phi_{A_{1}}^{(2)}\right)(\theta)=0$ permits one to define, in the case of $\left.\left(\Phi_{A_{0}}^{(1)}(\theta), \Phi_{B_{1}}^{(2)}(\theta)\right)_{\theta}\right|_{\Phi^{(1)}=\Phi^{(2)}=0} \neq 0$, a part of the superfields $\Lambda^{A_{0}}$ as functions of $\Gamma_{k}^{P}(\theta)$. A proof of consistency conditions for the constraints $\Phi_{A_{2}}^{(3)}(\theta)$ permits one to obtain new secondary antisymplectic constraints $\Phi_{A_{3}}^{(4)}(\theta)$ and a part from $\Lambda^{A_{0}}$, etc.

As a result of this algorithm, the complete set of $\bar{R}_{0}$ dependent antisymplectic constraints $\Phi_{I_{0}}\left(\Gamma_{k}(\theta), \theta\right)=0$ consisting of the primary $\Phi_{A_{0}}^{(1)}(\theta)$ and the remaining $\Phi_{A_{1 . .}}^{(2 .)}(\theta)$, called secondary ones, is defined as

$$
\Phi_{I_{0}}(\theta)=\left(\Phi_{A_{0}}^{(1)}, \Phi_{A_{1 . .}}^{(2 . .)}\right)(\theta), \Phi_{A_{1 . .}}^{(2 . .)}=\left(\Phi_{A_{1}}^{(2)}, \Phi_{A_{2}}^{(3)}, \ldots\right), I_{0}=1, \ldots, R_{0}^{(0)}, \ldots, R_{0}, \vec{\varepsilon}\left(\Phi_{I_{0}}\right)=\vec{\varepsilon}_{I_{0}}
$$

From the nonhomogeneous linear equations with unknowns $\Lambda^{A_{0}}(\theta)$, whose number is $R_{0}^{(1)}$,

$$
\partial_{\theta} \Phi_{I_{0}}(\theta)=\left(\mathbf{Q}_{\mathrm{compl}}^{(1)} \Phi_{I_{0}}\right)(\theta)=\left(\mathbf{Q}_{\mathrm{compl}} \Phi_{I_{0}}\right)(\theta)-\Lambda^{A_{0}}\left(\Phi_{A_{0}}^{(1)}(\theta), \Phi_{I_{0}}(\theta)\right)_{\theta} \approx 0
$$

with the symbol " $\approx$ " for a weak equality in view of Theorem $1, \mathcal{F}(\theta) \approx \mathcal{G}(\theta) \Leftrightarrow(\mathcal{F}-\mathcal{G})(\theta)=C^{I_{0}}\left(\Gamma_{k}(\theta)\right) \Phi_{I_{0}}(\theta)$, the consistency of Dirac's algorithm implies the existence of a general solution in the form

$$
\Lambda^{A_{0}}(\theta) \approx \Lambda_{\text {part }}^{A_{0}}\left(\Gamma_{k}(\theta), \theta\right)+\lambda_{0}^{a_{0}}(\theta) E_{a_{0}}^{A_{0}}\left(\Gamma_{k}(\theta), \theta\right), a_{0}=1, \ldots, r_{0} \leq R_{0}^{(0)}
$$

Here, $\Lambda_{\text {part }}^{A_{0}}(\theta)$ is a particular solution of the nonhomogeneous equations and the functions $E_{a_{0}}^{A_{0}}(\theta)$ define linearly independent solutions of homogeneous equations associated to (2.46), with $\left(\mathbf{Q}_{\text {compl }} \Phi_{I_{0}}\right)(\theta) \approx 0$ for $\operatorname{rank}_{\varepsilon_{J}}\left\|\left(\Phi_{A_{0}}^{(1)}(\theta), \Phi_{I_{0}}(\theta)\right)_{\theta}\right\| \Phi_{I_{0}}=0$ const[28]. The coefficients $\lambda_{0}^{a_{0}}(\theta)$ in Eqs. (2.47) are arbitrary.

Relations (2.47) allow one to rewrite HS (2.40) in terms of a total Hamiltonian action $\left.S_{\mathrm{H}}^{\mathrm{T}}\left(\left(\Gamma_{k}, \lambda\right)(\theta), \theta\right)\right)$,

$$
\partial_{\theta} \Gamma_{k}^{P}(\theta)=\left(S_{\mathrm{H}}^{\mathrm{T}}(\theta), \Gamma_{k}^{P}(\theta)\right)_{\theta}=\operatorname{ad} S_{\mathrm{H}_{0}}(\theta)\left(\Gamma_{k}^{P}(\theta)\right)+\lambda_{0}^{a_{0}}(\theta) \operatorname{ad} \Phi_{a_{0}}^{(1)}(\theta)\left(\Gamma_{k}^{P}(\theta)\right)=0,
$$

with $S_{\mathrm{H}_{0}}\left(\Gamma_{k}(\theta), \theta\right)=\left(S_{\mathrm{H}}+\Lambda_{\text {part }}^{A_{0}} \Phi_{A_{0}}^{(1)}\right)(\theta)$ and linearly dependent constraints $\Phi_{a_{0}}^{(1)}(\theta)=\left(E_{a_{0}}^{A_{0}} \Phi_{A_{0}}^{(1)}\right)(\theta)$, and proper zeroeigenvalue eigenvectors, $Z_{a_{1}}^{a_{0}}\left(\Gamma_{k}(\theta), \theta\right), a_{1}=1, \ldots, r_{1} \geq R_{0}^{(0)}-R_{0}^{\prime(1)}-r_{0}$ for a rigorous inequality being dependent as well.

Following Dirac's terminology, the concept of ( $\theta$-local) quantities of first and second classes is defined by the fact that an arbitrary $C^{\infty}\left(\Pi T^{*} \mathcal{M}_{k}\right)$-function $\mathcal{F}\left(\Gamma_{k}(\theta), \theta\right)$ either obeys the relations

$$
\left(\Phi_{I_{0}}(\theta), \mathcal{F}(\theta)\right)_{\theta} \approx 0 \Longleftrightarrow\left(\Phi_{I_{0}}(\theta), \mathcal{F}(\theta)\right)_{\theta}=g_{I_{0}}^{g_{0}}(\theta) \Phi_{g_{0}}(\theta)
$$

(and is said to be a first-class function) or is a second-class one if it does not obey Eqs. (2.49). Jacobi's identity for the antibracket $(\cdot, \cdot)_{\theta}$ implies that the set of first-class functions forms a Lie algebra $\mathcal{G}_{\text {I }}$ with a multiplication with respect to the antibracket, i.e., for any $\mathcal{F}(\theta), \mathcal{G}(\theta) \in \mathcal{G}_{\mathrm{I}},(\mathcal{F}(\theta), \mathcal{G}(\theta))_{\theta} \in \mathcal{G}_{\mathrm{I}}$. By definition, it follows that the total action $S_{\mathrm{H}}^{\mathrm{T}}(\theta)$ is the sum of certain quantities $S_{\mathrm{H}_{0}}(\theta)$ and $\left(\lambda_{0}^{a_{0}} \Phi_{a_{0}}^{(1)}\right)(\theta)$ in view of the non-uniqueness of the particular solution $\Lambda_{\text {part }}^{A_{0}}$ of Eqs. (2.46) which are given by the gauge algebra of reducible antisymplectic primary first-class constraints $\left[S_{\mathrm{H}_{0}}, \Phi_{a_{0}}^{(1)}\right](\theta)$ :

$$
\left(\Phi_{a_{0}}^{(1)}(\theta), \Phi_{a_{0}}^{(1)}(\theta)\right)_{\theta}=\mathbf{U}_{a_{0} b_{0}}^{c_{0}}(\theta) \Phi_{c_{0}}^{(1)}(\theta),\left(S_{\mathrm{H}_{0}}(\theta), \Phi_{a_{0}}^{(1)}(\theta)\right)_{\theta}=\mathbf{V}_{a_{0}}^{b_{0}}(\theta) \Phi_{b_{0}}^{(1)}(\theta),\left(\Phi_{a_{0}}^{(1)} Z_{a_{1}}^{a_{0}}\right)(\theta)=0
$$

with $\vec{\varepsilon}$-odd quantities $(\mathbf{U}, \mathbf{V})$ and $\vec{\varepsilon}$-even $Z$.

The primary constraints $\Phi_{a_{0}}^{(1)}(\theta)$ may be related with the reducible antisymplectic gauge transformations, $\delta^{\text {as }} \Gamma_{k}^{P}(\theta)$, in $C^{\infty}\left(\Pi T^{*} \mathcal{M}_{k}\right)$ (different from general gauge transformations, $\delta_{g} \mathcal{A}^{I}(\theta)=\int d \theta_{0} \hat{\mathcal{R}}_{\mathcal{H}_{0}}^{I}\left(\theta ; \theta_{0}\right) \xi^{\mathcal{A}_{0}}\left(\theta_{0}\right)$ ) which correspond to the difference between the values of $\Gamma_{k}^{P}(\theta)$ at $\theta$ and at $(\theta+\mu)$ under two different choices of the Lagrangian multipliers $\lambda_{0}^{a_{0}}(\theta), \tilde{\lambda}_{0}^{a_{0}}(\theta)$ : $\delta \lambda_{0}^{a_{0}}(\theta)=\left(\lambda_{0}^{a_{0}}-\tilde{\lambda}_{0}^{a_{0}}\right) \mu \equiv \mu^{a_{0}}(\theta)$ with arbitrary functions $\mu^{a_{0}}(\theta)$ on $\widetilde{M}$,

$$
\delta^{\mathrm{as}} \Gamma_{k}^{P}(\theta)=\delta \lambda_{0}^{a_{0}}(\theta)\left(\Phi_{a_{0}}^{(1)}(\theta), \Gamma_{k}^{P}(\theta)\right)_{\theta} \equiv \mu^{a_{0}}(\theta)\left(\Phi_{a_{0}}^{(1)}(\theta), \Gamma_{k}^{P}(\theta)\right)_{\theta}, \vec{\varepsilon}\left(\mu^{a_{0}}\right)=\vec{\varepsilon}_{a_{0}}+(1,0,1)
$$

For the choice $\mu^{a_{0}}(\theta)=\left(\mu^{a_{1}} Z_{a_{1}}^{a_{0}}\right)(\theta)$ with arbitrary functions $\mu^{a_{1}}(\theta)$ on $\tilde{M}$, being the Grassmann parities $\vec{\varepsilon}\left(\mu^{a_{1}}\right)=\vec{\varepsilon}_{a_{1}}+(1,0,1)$ if $\vec{\varepsilon}\left(Z_{a_{1}}^{a_{0}}\right)=\left(\vec{\varepsilon}_{a_{0}}+\vec{\varepsilon}_{a_{1}}\right)$, the above transformations vanish on the constraint surface, $\delta^{\text {as }} \Gamma_{k}^{P}(\theta) \approx 0$. 
The functional $Z_{\mathrm{H}}^{\mathrm{T}}\left[\Gamma_{k}, \lambda\right]$, given by

$$
Z_{\mathrm{H}}^{\mathrm{T}}\left[\Gamma_{k}, \lambda\right]=\int d \theta\left[V_{P}\left(\Gamma_{k}(\theta)\right) \overleftarrow{\partial}_{\theta} \Gamma_{k}^{P}(\theta)-S_{\mathrm{H}}^{\mathrm{T}}\left(\left(\Gamma_{k}, \lambda\right)(\theta), \theta\right)\right]
$$

is invariant not only with respect to the transformations $\delta_{g} \mathcal{A}^{I}(\theta)$, in view of the relation $Z_{\mathrm{H}}=Z_{\mathrm{H}}^{(1)}=Z_{\mathrm{H}}^{\mathrm{T}}$ and Eqs. (2.23), but also with respect to extended antisymplectic transformations in $C^{\infty}\left(\Pi T^{*} \mathcal{M}_{C L} \oplus \mathcal{M}_{\lambda}\right)$,

$$
\begin{aligned}
\delta \Gamma_{k}^{P}(\theta) & =-\mu^{a_{0}}(\theta)\left(\Phi_{a_{0}}^{(1)}(\theta), \Gamma_{k}^{P}(\theta)\right)_{\theta} \\
\delta \lambda_{0}^{a_{0}}(\theta) & =-\mu^{c_{0}}(\theta)\left(\delta_{c_{0}}^{a_{0}} \partial_{\theta}-\mathbf{V}_{c_{0}}^{a_{0}}(\theta)-\lambda_{0}^{b_{0}} \mathbf{U}_{c_{0} b_{0}}^{a_{0}}(\theta)(-1)\right)-\left(\mu^{a_{1}} Z_{a_{1}}^{a_{0}}\right)(\theta), \vec{\varepsilon}\left(\mu^{a_{1}}\right)=\vec{\varepsilon}_{a_{1}}
\end{aligned}
$$

Following an analog of "Dirac's conjecture" in the usual Hamiltonian treatment of a gauge model, one may suggest that the structure of $S_{\mathrm{L}}(\theta)$ for an LSM be such that secondary first-class antisymplectic constraints are generators of antisymplectic gauge transformations as well.

A characteristic feature of odd Hamiltonization is the possibility of an explicit identification of so-called antisymplectic gauge freedom in comparison with the odd-Lagrangian formulation of an LSM which is related to the construction of an extended Hamiltonian action $S_{\mathrm{H}}^{\mathrm{E}}(\theta)$ and a functional $Z_{\mathrm{H}}^{\mathrm{E}}$, including all the constraints $\Phi_{I_{0}}(\theta)$,

$$
Z_{\mathrm{H}}^{\mathrm{E}}\left[\Gamma_{k}, \Lambda\right]=\int d \theta\left[V_{P}\left(\Gamma_{k}(\theta)\right) \overleftarrow{\partial}_{\theta} \Gamma_{k}^{P}(\theta)-S_{\mathrm{H}}^{\mathrm{E}}\left(\left(\Gamma_{k}, \Lambda\right)(\theta), \theta\right)\right], S_{\mathrm{H}}^{\mathrm{E}}(\theta)=\left(S_{\mathrm{H}_{0}}+\Lambda^{I_{0}} \Phi_{I_{0}}\right)(\theta)
$$

The variational principle for $Z_{\mathrm{H}}^{\mathrm{E}}$ encodes an HS, which is not entirely equivalent to LS (2.3),

$$
\partial_{\theta} \Gamma_{k}^{P}(\theta)=\left(S_{\mathrm{H}}^{\mathrm{E}}(\theta), \Gamma_{k}^{P}(\theta)\right)_{\theta}=0, \Phi_{I_{0}}(\theta)=0 .
$$

The system of antisymplectic constraints $\left\{\Phi_{I_{0}}(\theta)\right\}$ contains two subsystems $\left\{\Phi_{I_{0}}\right\}=\left\{\Theta_{\hat{a}_{0}}, \Xi_{\tau_{0}}\right\}$ of first-class $\Theta_{\hat{a}_{0}}(\theta)$ and second-class $\Xi_{\tau_{0}}(\theta)$ constraints extracted from the initial system by means of certain quantities $g_{\hat{a}_{0}}^{I_{0}}(\theta): \Theta_{\hat{a}_{0}}(\theta)=\left(g_{\hat{a}_{0}}^{I_{0}} \Phi_{I_{0}}\right)(\theta)$ so that only the functions $\lambda^{\hat{0}_{0}}(\theta)$ from the set of Lagrangian multipliers $\Lambda^{I_{0}}(\theta), \Lambda^{I_{0}}(\theta)=\left(\lambda^{\hat{a}_{0}} g_{\hat{a}_{0}}^{I_{0}}\right)(\theta)$, remain completely undetermined by the evolution of HS (2.54).

By definition, we consider that an $\left(L_{1}^{\text {as }}, L_{2}^{\text {as }}\right)$-stage reducible system of antisymplectic constraints $\Phi_{I_{0}}(\theta)=\left(\Theta_{\hat{a}_{0}}, \Xi_{\tau_{0}}\right)(\theta)$ is divided to a subsystem of $L_{1}^{\text {as }}$-stage reducible first-class constraints $\left\{\Theta_{\hat{a}_{0}}(\theta)\right\}$ and that of $L_{2}^{\text {as }}$-stage reducible second-class ones $\left.\left\{\Xi_{\tau_{0}}\right)(\theta)\right\}$ for $\left(L_{1}^{\text {as }}, L_{2}^{\text {as }}\right) \in \mathbb{N}_{0}^{2}$ if

$$
\begin{aligned}
& \left(\Theta_{\hat{a}_{0}}(\theta), \Theta_{\hat{b}_{0}}(\theta)\right)_{\theta}=\left[U_{\hat{a}_{0} \hat{b}_{0}}^{\mathrm{I} \hat{c}_{0}} \Theta_{\hat{c}_{0}}+U_{\hat{a}_{0} \hat{b}_{0}}^{\mathrm{II} \Xi_{\tau_{0}} \sigma_{0}} \Xi_{\sigma_{0}}\right](\theta),\left(S_{\mathrm{H}_{0}}(\theta), \Theta_{\hat{a}_{0}}(\theta)\right)_{\theta}=\left[V_{\hat{a}_{0}}^{\mathrm{I} \hat{b}_{0}} \Theta_{\hat{b}_{0}}+V^{\mathrm{II} \tau_{0} \sigma_{0}} \Xi_{\hat{a}_{0}} \Xi_{\tau_{0}} \Xi_{\sigma_{0}}\right](\theta), \\
& \left(\Xi_{\tau_{0}}(\theta), \Xi_{\sigma_{0}}(\theta)\right)_{\theta}=\left[E_{\tau_{0} \sigma_{0}}+U_{\tau_{0} \sigma_{0}}^{\mathrm{II} \rho_{0}} \Xi_{\rho_{0}}\right](\theta),\left(S_{\mathrm{H}_{0}}(\theta), \Xi_{\tau_{0}}(\theta)\right)_{\theta}=\left[V^{\mathrm{II} \sigma_{0}} \Xi_{\tau_{0}} \Xi_{\sigma_{0}}\right](\theta),
\end{aligned}
$$

where $\left\|E_{\tau_{0} \sigma_{0}}\right\|_{\Phi_{I_{0}}=0} \neq\left\|0_{\tau_{0} \sigma_{0}}\right\|, \hat{a}_{0}, \hat{b}_{0}, \hat{c}_{0}=1, \ldots, r_{0}^{\mathrm{I}} \leq R_{0}, \tau_{0}, \sigma_{0}=1, \ldots, R_{0}-r_{0}^{\mathrm{I}} \equiv r_{0}^{\mathrm{II}}$ and

$$
\begin{aligned}
& \operatorname{rank}\left\|\left(\Theta_{\hat{a}_{0}}, \Gamma_{\mathrm{k}}^{P}(\theta)\right)_{\theta} \omega_{P Q}^{k}\right\|_{\Phi_{I_{0}}=0}=\bar{r}^{\mathrm{I}}<\bar{r}_{0}^{\mathrm{I}}, \operatorname{rank}\left\|\left(\Xi_{\tau_{0}}(\theta), \Gamma_{\mathrm{k}}^{P}(\theta)\right)_{\theta} \omega_{P Q}^{k}\right\|_{\Phi_{I_{0}}=0}=\bar{r}^{\mathrm{II}}<\bar{r}_{0}^{\mathrm{II}} \\
& \left(\Theta_{\hat{a}_{0}} Z_{\hat{a}_{1}}^{\mathrm{a} \hat{a}_{0}}\right)(\theta)=0,\left(Z_{\hat{a}_{s-1}}^{\hat{a}_{s-2}} Z_{\hat{a}_{s}}^{\mathrm{I} \hat{a}_{s-1}}\right)(\theta)=\Theta_{\hat{b}_{0}} L_{\hat{a}_{s}}^{\hat{a}_{s-2} \hat{b}_{0}}, s=1, \ldots, L_{1}^{\mathrm{as}}, \hat{a}_{s}=1, \ldots, r_{s}^{\mathrm{I}}, \\
& \operatorname{rank}\left\|Z_{\hat{a}_{s-1}}^{\hat{a}_{s-2}}(\theta)\right\|_{\Theta=0}=\sum_{k=0}^{s-1}(-1)^{k} \bar{r}_{s-k-2}^{\mathrm{I}}<\bar{r}_{s-1}^{\mathrm{I}}, \operatorname{rank} \| Z_{L_{1}^{\mathrm{as}}}^{\mathrm{I}_{\mathrm{a}_{1}}^{\hat{L}_{1}^{\mathrm{as}}-1}(\theta) \|_{\Theta=0}=\sum_{k=0}^{L_{1}^{\mathrm{as}}-1}(-1)^{k} \bar{r}_{L_{1}^{\mathrm{I}} \mathrm{I}}^{\mathrm{I}}-k-1}=\bar{r}_{L_{1}^{\mathrm{as}}}^{\mathrm{I}}, \\
& (\vec{\varepsilon}, \mathrm{gh}) Z_{\hat{a}_{s}}^{\hat{a}_{s-1}}=\left(\vec{\varepsilon}_{\hat{a}_{s-1}}+\vec{\varepsilon}_{\hat{a}_{s}},-\mathrm{gh}_{\hat{a}_{s-1}}+\mathrm{gh}_{\hat{a}_{s}}\right)\left(Z_{\hat{a}_{0}}^{\hat{a}_{-1}}, L_{\hat{a}_{1}}^{\hat{a}_{-1} \hat{b}_{0}}, r_{-1}^{\mathrm{I}}\right) \equiv\left(\Theta_{\hat{a}_{0}}, 0, r^{\mathrm{I}}\right),
\end{aligned}
$$

where we have used the standard distribution $[1,3]$ of ghost number in $\Pi T^{*} \mathcal{M}_{\mathrm{CL}}$.

A complete definition of the subsystem of second-class antisymplectic constraints $\left\{\Xi_{\tau_{0}}(\theta)\right\}$ may be obtained directly from Eqs. (2.57) under the change $\left(\Theta_{\hat{a}_{0}}, Z_{\hat{a}_{s}}^{\hat{a}_{s-1}}, L_{\hat{a}_{s}}^{\hat{a}_{s-2} \hat{b}_{0}}, r_{s}^{\mathrm{I}}, L_{1}^{\mathrm{as}}\right) \rightarrow\left(\Xi_{\tau_{0}}, Z_{\tau_{s}}^{\mathrm{II} \tau_{s-1}}, L_{\tau_{s}}^{\tau_{s-2} \sigma_{0}}, r_{s}^{\mathrm{II}}, L_{2}^{\mathrm{as}}\right)$. For $L_{1}^{\text {as }}=0\left(L_{2}^{\text {as }}=0\right)$, the subsystem $\left\{\Theta_{\hat{a}_{0}}(\theta)\right\}\left(\left\{\Xi_{\tau_{0}}(\theta)\right\}\right)$, is referred to as irreducible first-class (second-class) antisymplectic constraints. The definitions in Eqs. (2.55)-(2.58) generalize, to the case of reducible mixed antisymplectic constraints in the context of a dynamical LSM, the formal definition $[6,23]$ of irreducible second-class antisymplectic constraints for $\theta=0$. 
The presence of the reducible constraints $\left\{\Xi_{\tau_{0}}\right\}$ permits one to construct a so-called weak Dirac's antibracket possessing all the properties of the odd Poisson bracket on the constraint surface $\Xi_{\tau_{0}}=0$ by means of a degenerate odd Poisson-Dirac bivector:

$$
\omega_{k D}^{P Q}(\theta)=\left(\Gamma_{k}^{P}(\theta), \Gamma_{k}^{Q}(\theta)\right)_{\theta D}=\omega_{k}^{P S}(\theta)\left(\omega_{S T}^{k}(\theta)-\frac{\vec{\partial} \Xi_{\tau_{0}}(\theta)}{\partial \Gamma_{k}^{S}(\theta)} \widetilde{E}^{\tau_{0} \sigma_{0}}(\theta) \frac{\overleftarrow{\partial} \Xi_{\sigma_{0}}(\theta)}{\partial \Gamma_{k}^{T}(\theta)}\right) \omega_{k}^{T Q}(\theta)
$$

with the quantities $\widetilde{E}^{\tau_{0} \sigma_{0}}\left(\Gamma_{k}(\theta)\right)$ determined on the surface $\Xi_{\tau_{0}}=0,\left(A\left(\Gamma_{k}(\theta)\right), \Xi_{\tau_{0}}(\theta)\right)_{\theta D} \approx 0$, for any $A\left(\Gamma_{k}(\theta)\right) \in C^{\infty}\left(\Pi T^{*} \mathcal{M}_{k}\right)$, characterizing the Dirac antibracket, by the equations

$$
\widetilde{E}^{\tau_{0} \sigma_{0}}(\theta)\left(\Xi_{\sigma_{0}}(\theta), \Xi_{\rho_{0}}(\theta)\right)_{\theta}=\delta^{\tau_{0}} \rho_{0}-\left(Z^{\mathrm{II}} \tau_{\tau_{1}} d_{\sigma_{0}}^{\tau_{1}}\right)(\theta)
$$

The functions $d_{\sigma_{0}}^{\tau_{1}}\left(\Gamma_{k}(\theta)\right), \vec{\varepsilon}\left(d_{\sigma_{0}}^{\tau_{1}}\right)=\vec{\varepsilon}_{\tau_{1}}+\vec{\varepsilon}_{\sigma_{0}}$ in (2.60) may be specified due to the consequence $\left(E_{\tau_{0} \sigma_{0}} Z^{\text {II } \sigma_{0}}\right)(\theta) \approx 0$ of relations (2.56), (2.58), applied to the constraints $\Xi_{\tau_{0}}(\theta)$ in the form $\left(Z_{\tau_{1}}^{\mathrm{II} \tau_{0}} d_{\tau_{0}}^{\sigma_{1}}\right)(\theta) \approx \delta_{\tau_{1}}^{\sigma_{1}}$.

The fact that Dirac's antibracket obeys the generalized Jacobi identity in the entire $\Pi T^{*} \mathcal{M}_{k}$ or on the surface $\Xi_{\tau_{0}}(\theta)=0$ can be extended according to the construction of a weak even Dirac bracket for infinitely reducible second-class symplectic constraints [24] used to quantize the $N=1$ Brink-Schwarz superparticle and the $N=1, d=9$ massive superparticle with the Wess-Zumino term.

As in the case of the initial antibracket, there exists an odd weak nilpotent (on $\Xi_{\tau_{0}}(\theta)=0$ ) Laplacian $\Delta_{D}^{k}(\theta)$, corresponding to antibracket $(\cdot, \cdot)_{\theta D}$, such that Dirac's antibracket weakly equals to the failure of $\Delta_{D}^{k}(\theta)$ to act as a derivative on the product of two functions in $C^{\infty}\left(\Pi T^{*} \mathcal{M}_{k}\right)$,

$$
\Delta_{D}^{k}(\theta)=\frac{(-1)^{\varepsilon\left(\Gamma^{P}\right)}}{2} \rho_{D}^{-1} \frac{\vec{\partial}}{\partial \Gamma_{k}^{P}(\theta)}\left(\rho_{D} \omega_{k D}^{P Q}\right)_{k}(\theta) \frac{\vec{\partial}}{\partial \Gamma_{k}^{Q}(\theta)}=\frac{(-1)^{\varepsilon\left(\Gamma^{Q}\right)}}{2} \rho_{D}^{-1} \omega_{Q P}^{k}(\theta)\left(\Gamma_{k}^{P}(\theta), \rho_{D}\left(\Gamma_{k}^{Q}(\theta), \cdot\right)_{\theta D}\right)_{\theta}
$$

with an $\vec{\varepsilon}$-even density function $\rho_{D}\left(\Gamma_{k}(\theta)\right)$. The operator $\Delta_{D}^{k}(\theta)$ generalizes the properties of its analogue for irreducible antisymplectic second-class constraints in Ref. [6], for $U_{\tau_{0} \sigma_{0}}^{\mathrm{II} \rho_{0}}=0$ in Eqs. (2.56).

From the definition of Dirac's antibracket and relations (2.56)-(2.59), there follows, for any first-class $C^{\infty}\left(\Pi T^{*} \mathcal{M}_{k}\right)$-functions $[\mathcal{F}, \mathcal{G}](\theta)$ and arbitrary $C^{\infty}\left(\Pi T^{*} \mathcal{M}_{k}\right)$-function $\mathcal{R}(\theta)$, the validity of the weak equalities

$$
(\mathcal{F}(\theta), \mathcal{G}(\theta))_{\theta D} \approx(\mathcal{F}(\theta), \mathcal{G}(\theta))_{\theta},\left(\mathcal{F}(\theta),(\mathcal{G}(\theta), \mathcal{R}(\theta))_{\theta D}\right)_{\theta D} \approx\left(\mathcal{F}(\theta),(\mathcal{G}(\theta), \mathcal{R}(\theta))_{\theta}\right)_{\theta} .
$$

By virtue of the fact that $S_{\mathrm{H}}^{\mathrm{E}}(\theta)$ is a first-class quantity, the Dirac antibracket generates the odd HS (2.54), where it is possible to change the antibracket $(\cdot, \cdot)_{\theta}$ by $(\cdot, \cdot)_{\theta D}$. In turn, the definition of the first-class constraint subsystem (2.55), with allowance for relations (ref2.61), may be rewritten in terms of Dirac's antibracket with a weak equality which means an equality modulo $\Xi_{\tau_{0}}(\theta)$. Note that in view of the inequality $Z_{\mathrm{H}}^{\mathrm{T}} \neq Z_{\mathrm{H}}^{\mathrm{E}}$, the functional $Z_{\mathrm{H}}^{\mathrm{E}}$ is not invariant under the general gauge transformations $\delta_{g} \mathcal{A}^{I}(\theta)$ due to the presence of the secondary constraints $\Phi_{A_{1 . .}}^{2 . .}(\theta)$.

\section{ODD-LAGRANGIAN FORM OF BFV-BRST METHOD APPLICATION TO ODD-HAMILTONIAN LSM}

Let us apply the BFV-BRST method [11] to an LSM in the odd Hamiltonian formulation which has been made more complex by the presence of the $\left(L_{1}^{\mathrm{as}}, L_{2}^{\mathrm{as}}\right)$-reducible first- and second-class antisymplectic constraints. The construction of an analog $S_{\Omega}(\theta)$ of the BFV-BRST charge, encoding, in terms of Dirac's antibracket, the gauge algebra structure functions of the antisymplectic first-class constraints $\Theta_{\hat{a}_{0}}$ and the eigenvectors $Z_{\hat{a}_{s}}^{\mathrm{I} \hat{a}_{s-1}}, s=0, \ldots, L_{1}^{\text {as }}$, as well as the enhanced antisymplectic gauge transformations, can be described by a superfield algorithm similar to the construction of the superfield BV action in Refs. [1, 4]. Let us consider the gauge transformations (2.52) restricted in the minimal sector of superfields $\left\{\Gamma_{k}^{P}(\theta)\right\}=\Pi T^{*} \mathcal{M}_{k}$ for all first-class constraints $\Theta_{\hat{a}_{0}}$,

$$
\delta^{\mathrm{as}} \Gamma_{k}^{P}(\theta)=\left(\Gamma_{k}^{P}(\theta), \Theta_{\hat{a}_{0}}(\theta)\right)_{\theta D} \mu^{\hat{a}_{0}}(\theta),(\vec{\varepsilon}, \mathrm{gh}) \mu^{\hat{a}_{0}}=\left(\vec{\varepsilon}_{\hat{a}_{0}}+(1,0,1),-1-\mathrm{gh}_{\hat{a}_{0}}\right), \operatorname{gh}_{\hat{a}_{0}} \equiv \operatorname{gh}\left(\Theta_{\hat{a}_{0}}\right)
$$


which, due to the definition $\delta^{\mathrm{as}} \Gamma_{k}^{P}(\theta)=\Gamma_{k}^{P}(\theta+\mu)-\Gamma_{k}^{P}(\theta)=\overleftarrow{\partial}_{\theta} \Gamma_{k}^{P}(\theta) \mu,(\vec{\varepsilon}, \mathrm{gh}) \mu=((1,0,1),-1)$ and the substitution, instead of arbitrary $\mu^{\hat{a}_{0}}(\theta), \mu^{\hat{a}_{0}}(\theta)=d \widetilde{\mu}^{\hat{a}_{0}}(\theta)$, of the ghosts $d \widetilde{\mu}^{\hat{a}_{0}}(\theta)=C^{\hat{a}_{0}}(\theta) d \theta$, $(\vec{\varepsilon}, \mathrm{gh}) C^{\hat{a}_{0}}=\left(\vec{\varepsilon}_{\hat{a}_{0}},-\mathrm{gh}_{\hat{a}_{0}}\right)$, are embedded into the odd HS in $\Pi T^{*} \mathcal{M}_{k}$ with $2 N$ equations (for $\mu=d \theta$ )

$$
\overleftarrow{\partial}_{\theta} \Gamma_{k}^{P}(\theta) \approx\left(\Gamma_{k}^{P}(\theta), S_{\Omega 1}^{0}(\theta)\right)_{\theta D}, S_{\Omega 1}^{0}(\theta)=\left(\Theta_{\hat{a}_{0}} C^{\hat{a}_{0}}\right)(\theta),(\vec{\varepsilon}, \mathrm{gh}) S_{\Omega 1}^{0}(\theta)=(\overrightarrow{0}, 0)
$$

In view of (2.58), the function $S_{\Omega_{1}}^{0}(\theta)$ is invariant, modulo $\Xi_{\tau_{0}}(\theta)$, with respect to antisymplectic gauge transformations of ghosts $C^{\hat{a}_{0}}(\theta)$, with arbitrary functions $\mu^{\hat{a}_{1}}(\theta) \in C^{\infty} \mathcal{M},(\vec{\varepsilon}, \mathrm{gh}) \mu^{\hat{a}_{1}}=\left(\vec{\varepsilon}_{\hat{a}_{1}},-\mathrm{gh}_{\hat{a}_{1}}\right)$,

$$
\delta^{\mathrm{as}} C^{\hat{a}_{0}}(\theta)=\left(C^{\hat{a}_{0}}(\theta), \Theta_{\hat{a}_{1}}(\theta)\right)_{\theta D} \mu^{\hat{a}_{1}}(\theta), \Theta_{\hat{a}_{1}}=C_{\hat{a}_{0}}^{*} Z_{\hat{a}_{1}}^{\mathrm{l} \hat{a}_{0}},(\vec{\varepsilon}, \mathrm{gh}) C_{\hat{a}_{0}}^{*}=\left(\vec{\varepsilon}_{\hat{a}_{0}}+(1,0,1), \mathrm{gh}_{\hat{a}_{0}}-1\right)
$$

Making the substitution $\mu^{\hat{a}_{1}}(\theta)=d \widetilde{\mu}^{\hat{a}_{1}}(\theta)=C^{\hat{a}_{1}}(\theta) d \theta$ and extending $r_{0}^{\mathrm{I}}$ first-order equations in $\theta$ with respect to unknown $C^{\hat{a}_{0}}(\theta)$ in transformations (3.3) to an HS-like set of $2 r_{0}^{\mathrm{I}}$ equations with the even Hamiltonian $S_{\Omega_{1}^{1}}^{1}\left(\Gamma_{k}, C_{0}^{*}, C_{1}\right)=\left(C_{\hat{a}_{0}}^{*} Z_{\hat{a}_{1}}^{\mathrm{I} \hat{a}_{0}} C^{\hat{a}_{1}}\right)(\theta)$ for unknown $\overleftarrow{\partial}_{\theta} \Gamma_{0}^{P_{0}}(\theta), \Gamma_{0}^{P_{0}}=\left(C^{\hat{a}_{0}}, C_{\hat{a}_{0}}^{*}\right)$, we obtain a system of the form (3.2). An extension of the union of the latter HS with Eqs. (3.2) is formally identical to the system (3.2) under the replacement

$$
\left(\Gamma_{0}^{P_{0}}, S_{\Omega_{1}}^{0}(\theta)\right) \rightarrow\left(\Gamma_{[0]}^{P_{[0]}}, S_{\Omega_{[1]}}^{1}\right):\left\{\Gamma_{[0]}^{P_{[0]}}=\left(\Gamma_{k}^{P}, \Gamma_{0}^{P_{0}}\right), \Gamma_{1}^{p_{1}}=\left(C^{\alpha_{1}}, \mathcal{C}_{\alpha_{1}}^{*}\right), S_{\Omega_{[1]}}^{1}=S_{\Omega_{1}^{0}}^{0}+S_{\Omega_{1}}^{1}\right\}
$$

The iteration sequence related to a reformulation of the antisymplectic gauge transformations of ghost variables $C^{\hat{a}_{0}}, \ldots, C^{\hat{a}_{s-2}}$ obtained from relations (2.58), leads, for an $L_{1}^{\text {as }}$-stage reducible LSM at the $s$-th step, with $0<s \leq L$ and $\Gamma_{C L}^{P} \equiv \Gamma_{-1}^{P_{-1}}$, to invariance transformations for $S_{\Omega_{1}^{s-1}}^{s}(\theta)$, modulo the constraints $\Theta_{\hat{a}_{0}}$, namely,

$$
\begin{aligned}
& \delta^{\mathrm{as}} C^{\hat{a}_{s-1}}(\theta)=\left(C^{\hat{a}_{s-1}}(\theta), \Theta_{\hat{a}_{s}}(\theta)\right)_{\theta D} \mu^{\hat{a}_{s}}(\theta),\left[\Theta_{\hat{a}_{s}}, S_{\Omega_{1}}^{s-1}\right](\theta)=\left[C_{\hat{a}_{s-1}}^{*} Z_{\hat{a}_{s}}^{\mathrm{I} \hat{a}_{s-1}}\left(\Gamma_{k}\right), \Theta_{\hat{a}_{s-1}} C^{\hat{a}_{s-1}}\right](\theta), \\
& (\vec{\varepsilon}, \mathrm{gh})\left[\mu^{\hat{a}_{s}}, S_{\Omega_{1}}^{s-1}\right]=\left[\left(\vec{\varepsilon}_{\hat{a}_{s}}+(s-1)(1,0,1),-\mathrm{gh}_{\hat{a}_{s}}+s-1\right),(\overrightarrow{0}, 0)\right] .
\end{aligned}
$$

The substitution $\mu^{\hat{a}_{s}}(\theta)=d \widetilde{\mu}^{\hat{a}_{s}}(\theta)=C^{\hat{a}_{s}}(\theta) d \theta$ transforms the antisymplectic gauge transformations (3.4) to $r_{s-1}$ equations for unknown $C^{\hat{a}_{s-1}}(\theta)$, extended by the superantifields of odd momenta $C_{\hat{a}_{s-1}}^{*}(\theta)$, into an HS on $\Pi T^{*} \mathcal{M}_{s-1}=\left\{\Gamma_{s-1}^{P_{s-1}}\right\}$ :

$$
\overleftarrow{\partial}{ }_{\theta} \Gamma_{s-1}^{P_{s-1}}(\theta)=\left(\Gamma_{s-1}^{P_{s-1}}(\theta), S_{\Omega 1}^{s}(\theta)\right)_{\theta D}, S_{\Omega 1}^{s}(\theta)=\left(C_{\hat{a}_{s-1}}^{*} Z_{\hat{a}_{s}}^{\left[\hat{a}_{s-1}\right.}\left(\Gamma_{k}\right) C^{\hat{a}_{s}}\right)(\theta), \Gamma_{s-1}^{P_{s-1}}=\left(C^{\hat{a}_{s-1}, C_{\hat{a}_{s-1}}^{*}}\right)
$$

Having combined the system (3.5) with an HS in $\Pi T^{*} \mathcal{M}_{[s-1]}=\left\{\Gamma_{[s-1]}^{P_{[s-1]}}\right\}$ of the same form with $\overleftarrow{\partial}_{\theta} \Gamma_{[s-1]}^{P_{[s-1]}}(\theta)$ and the even BFV-BRST charge (Hamiltonian) $S_{\Omega[1]}^{s-1}(\theta)=\sum_{r=0}^{s-1} S_{\Omega_{[1]}^{r}}^{r}(\theta)$ and having expressed the result for $2\left(N+\sum_{l=0}^{s} r_{l}\right)$ equations with $S_{\Omega_{[1]}}^{s-1}(\theta)=\left(S_{\Omega_{[1]}^{s-1}}+S_{\Omega_{1}^{s}}^{s}\right)(\theta)$, we obtain, by induction, the following HS:

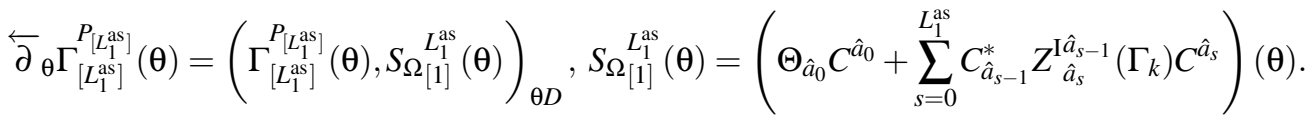

The even function $S_{\Omega_{[1]}}^{L_{1}^{\mathrm{as}}}(\theta)$ quadratic in the powers of $\left.\Theta_{\hat{a}_{0}}, \Gamma_{\left[L_{1}^{\mathrm{as}}\right]}^{P_{\left[L^{\mathrm{as}}\right]}} \equiv \Gamma_{k}^{P_{k}}(\theta)=\left(\Phi^{\mathcal{A}_{k}}, \Phi_{\mathcal{A}_{k}}^{*}\right)(\theta), \mathcal{A}_{k}=1, \ldots, N+\sum_{l=0}^{L_{1}^{\mathrm{as}}} r_{l}, k=\mathrm{MIN}\right\}$ with vanishing ghost number provided by the $(\vec{\varepsilon}, \mathrm{gh})$-spectrum for $\Pi T^{*} \mathcal{M}_{k}$-coordinates

$$
(\vec{\varepsilon}, \mathrm{gh}) C^{\hat{a}_{s}}=\left(\vec{\varepsilon}_{\hat{a}_{s}}+s(1,0,1),-\mathrm{gh}_{\hat{a}_{s}}+s\right),(\vec{\varepsilon}, \mathrm{gh}) \Phi_{\mathcal{A}_{k}}^{*}=\left(\vec{\varepsilon}\left(\Phi^{\mathcal{A}_{k}}\right)+(1,0,1),-1-\operatorname{gh}\left(\Phi^{\mathcal{A}_{k}}\right)\right),
$$

is a solution of the classical master equation written in terms of Dirac's antibracket trivially extended in $\Pi T^{*} \mathcal{M}_{k}=\left\{\Gamma_{k}^{p_{k}}(\theta)\right.$, $k=\mathrm{MIN}\}$ with accuracy up to $O\left(C^{\hat{a}_{s}}\right)$, modulo $\Phi_{I_{0}}(\theta)$,

$$
\left(S_{\Omega_{[1]}}^{L_{1}^{\mathrm{as}}}(\theta), S_{\Omega_{[1]}}^{L_{1}^{\mathrm{as}}}(\theta)\right)_{\theta D} \approx O\left(C^{\hat{a}_{s}}\right) .
$$

Additionally, the function $S_{\Omega[1]}{ }_{1}^{\mathrm{as}}(\theta)$ is subject to the BFV-like condition of properness in the sense that

$$
\begin{aligned}
& \operatorname{rank}\left\|\frac{\vec{\partial}}{\partial \Gamma_{k}^{P_{k}}(\theta)} \frac{\overleftarrow{\partial} S_{\Omega_{[1]}}^{L^{\mathrm{as}}}(\theta)}{\partial \Gamma_{k}^{Q_{k}}(\theta)}\right\|{ }_{\frac{\partial}{S_{\Omega}}=0}=2 \sum_{s=0}^{\left[\frac{L_{1}^{\mathrm{as}}+1}{2}\right]} \bar{r}_{2 s-\left(L_{1}^{\mathrm{as}} \bmod 2\right)}^{\mathrm{I}}=\bar{L}_{1}, \\
& L_{1}=\frac{1}{2}\left(\operatorname{codim}_{+}+\operatorname{codim}_{-}\right) \Pi T^{*} \Sigma_{1}^{\mathrm{as}}+\frac{1}{2} \sum_{s=0}^{L_{1}^{\mathrm{as}}}\left(\operatorname{dim}_{+}+\operatorname{dim}_{-}\right) \Pi T^{*} \mathcal{M}_{s},
\end{aligned}
$$


where the codimension of the surface $\Sigma_{1}^{\mathrm{as}}=\left\{\Gamma_{\mathrm{CL}}^{P}(\theta) \mid \Theta_{\hat{a}_{0}}(\theta)=0\right\}$ is calculated with respect to $\Pi T^{*} \mathcal{M}_{\mathrm{CL}}$. The integrability of the HS in (3.8) is guaranteed by a double deformation of $S_{\Omega_{[1]}}^{L_{1}^{\text {as }}}(\theta)$ : first in the powers of $\Phi_{\mathcal{A}_{k}}^{*}(\theta)$ and then in the powers of $C^{\hat{a}_{s}}(\theta)$, in the framework of the existence theorem [3] for the classical master equation in the minimal sector, in the case of a purely topological theory (i.e., one without the potential term $S_{0}(A)=S(\mathcal{A}(0), 0)$ in Sec. 2.1 presenting the classical action of a standard gauge model for $\left.\left(\varepsilon_{P}\right)_{I}=\left(\varepsilon_{P}\right)_{I}=\left(\varepsilon_{P}\right)_{\hat{a}_{s}}=0\right)$ :

$$
\left(S_{\Omega ; k}\left(\Gamma_{k}(\theta)\right), S_{\Omega ; k}\left(\Gamma_{k}(\theta)\right)\right)_{\theta D} \approx 0,(\vec{\varepsilon}, \mathrm{gh}) S_{\Omega ; k}=(\overrightarrow{0}, 0), k=\mathrm{MIN} .
$$

Theorem 3 : There exists a solution of equation (3.9), in $\Pi T^{*} \mathcal{M}_{\mathrm{MIN}}$, as a power series in the ghost coordinates $\Phi_{\mathrm{gh}}^{\hat{a}}(\theta)=$ $\left\{C^{\hat{a}_{s}}(\theta), s=0, \ldots, L_{1}^{a s}\right\}$, subject to the boundary conditions

$$
\left[S_{\Omega ; k}(\theta),\left(S_{\Omega ; k}(\theta), C_{\hat{a}_{0}}^{*}(\theta)\right)_{\theta D},\left(\left(C^{\hat{a}_{s-1}}(\theta), S_{\Omega ; k}(\theta)\right)_{\theta D}, C_{\hat{a}_{s}}^{*}(\theta)\right)_{\theta D}\right] \Phi_{\mathrm{gh}}^{\hat{a}}=0=\left[0, \Theta_{\hat{a}_{0}}, Z_{\hat{a}_{s}}^{\mathrm{I} \hat{a}_{s-1}}\right](\theta),
$$

and to the condition of properness (3.8) written for $S_{\Omega ; k}(\theta)$.

A sketch of a proof: the proof of Theorem 3 repeats the Koszul-Tate construction in Hamiltonian formalism [15] for the antisymplectic case.

\section{CONCLUSION}

Let us summarize the main results of the present work.

We have examined a $\theta$-local description of an arbitrary degenerate reducible superfield theory as a natural extension of a usual gauge theory, defined on a configuration space $\left.\mathcal{M}_{\mathrm{cl}}\right|_{\theta=0}$ of classical fields $A^{i}$, to a local superfield model. Namely, we apply Dirac's algorithm to realize an odd Hamiltonization of a Lagrangian degenerate local superfield model (LSM), being an extension of a usual gauge model of classical fields $A^{i}, i=$ $1, \ldots, n=n_{+}+n_{-}$, on a configuration space $M_{\mathrm{cl}}$, to a $\theta$-local theory defined on an odd tangent bundle $T_{\text {odd }} \mathcal{M}_{\mathrm{CL}} \equiv \Pi T \mathcal{M}_{\mathrm{CL}}$ $=\left\{\mathcal{A}^{I}, \partial_{\theta} \mathcal{A}^{I}\right\}, I=1, \ldots, N=N_{+}+N_{-},\left(n_{+}, n_{-}\right) \leq\left(N_{+}, N_{-}\right)$ with $N_{+},\left(N_{-}\right)$bosonic (fermionic) superfields. The generalized classical superfields $\mathcal{A}^{I}(\theta), \mathcal{A}^{I}(\theta)=A^{I}+\lambda^{I} \theta$, parameterize the base $\mathcal{M}_{\mathrm{CL}}\left(\mathcal{M}_{\mathrm{cl}} \subset \mathcal{M}_{\mathrm{CL}}\right)$ of the bundle $\Pi T \mathcal{M}_{\mathrm{CL}}$ and transform with respect to a $J$-superfield representation $T$ of the direct product of supergroups $\bar{J}, P: J=\bar{J} \times P, P=$ $\exp \left(i \mu p_{\theta}\right)$, with $\bar{J}$ chosen as a spacetime SUSY group, and $\mu$, $p_{\theta}$ being the respective nilpotent parameter and generator of $\theta$-shifts. The non-Lorentz character of superfields $\mathcal{A}^{I}(\theta)$ defined on $\mathcal{M}=\left\{\left(z^{M}, \theta\right)\right\}=\left\{z^{K}\right\}, z^{M} \subset i \subset I$, is reflected by a possible inclusion in their spectrum of additional, besides $\mathcal{A}^{i}(\theta)$, superfields corresponding to the ghosts of the minimal sector in the BV quantization scheme. Following the BFV prescription, we construct their $\theta$-local counterparts on the basis of a complete system of antisymplectic constraints and a Hamiltonian action $S_{\mathrm{H}_{0}}(\theta)$ defined on $\Pi T^{*} \mathcal{M}_{\mathrm{CL}}=\left\{\mathcal{A}^{I}, \mathcal{A}_{I}^{*}\right\}$, a bosonic BRST charge $S_{\Omega}(\theta)$, a unitarizing Hamiltonian action $S_{\mathcal{H}}(\theta)$, and a gauge fermion $F_{\Psi}(\theta)$. We specify to the case of a singular LSM a derivation of Lagrangian and Hamiltonian master equations from Noether's first theorem applied to $\theta$-shifts, and establish a relation between the complete Hamiltonian action, $S_{\mathrm{H}}(\theta)=S_{\mathcal{H}}(\theta)+\left(S_{\Omega}(\theta), F_{\Psi}(\theta)\right)_{\theta D}$, constructed via $\theta$-local Dirac's antibracket, and the quantum action of the BV method.

We have constructed an odd-Hamiltonian formulation for an LSM starting from an odd Lagrangian in the case of a degenerate Hessian supermatrix $\left\|\left(S_{\mathrm{L}}^{\prime \prime}\right)_{I J}\right\|(\theta)$ as the supermatrix of second derivatives of the Lagrangian classical action, $S_{\mathrm{L}}(\theta)=S_{\mathrm{L}}\left(\mathcal{A}(\theta), \partial_{\theta} \mathcal{A}(\theta), \theta\right)$, with respect to odd velocities $\left(\partial_{\theta} \mathcal{A}^{I}, \partial_{\theta} \mathcal{A}^{J}\right)(\theta)$ on the basis of Dirac's algorithm in terms of a $\theta$-local antibracket. We apply the BFV method to a construction of formal counterparts of the BFV-BRST charge, gauge fermion and unitarizing Hamiltonian of a $t$-local field theory in terms of a $\theta$-local Dirac's antibracket, reflecting, in view of general gauge invariance, the presence of a subsystem of second-class constraints among all of the antisymplectic constraints. We present a plan of establishing a correspondence between the resulting odd-Hamiltonian formulation of an LSM with the BV quantum action for a gauge model corresponding to an LSM.

Acknowledgments D.M.G. thanks the foundations FAPESP and CNPq for permanent support. P.Yu.M. is grateful to $\mathrm{CNPq}$.
[1] D.M. Gitman, P.Yu. Moshin, and A.A. Reshetnyak, J. Math. Phys. 46, 072302 (2005); Phys. Lett. B 621, 295 (2005).

[2] A.A. Reshetnyak, Basic features of general superfield quantization method for gauge theories in Lagrangian formalism, Proceedings of the International Seminar on Supersymmetries and Quantum Symmetries SQS 03, Dubna, Russia, July 24-29,
2003 (Eds. E. Ivanov and A. Pashnev, JINR, Dubna, 2004, 345); hep-th/0312118.

[3] I.A. Batalin and G.A. Vilkovisky, Phys. Lett. B 102, 27 (1981); Phys. Rev. D 28, 2567 (1983); J. Math. Phys. 26, 172 (1985).

[4] A.A. Reshetnyak, Russ. Phys. J. 47, 1008 (2004); hepth/0512327. 
[5] I.A. Batalin and I.V. Tyutin, Int. J. Mod. Phys. A 8, 2333 (1993).

[6] I.A. Batalin, K. Bering, and P.H. Damgaard, Nucl. Phys. B 739, 389 (2006); hep-th/0512131.

[7] J. Thierry-Mieg, J. Math. Phys. 21, 2834 (1980); L. Bonora and M. Tonin, Phys. Lett. B 98, 48 (1981); L. Bonora, P. Pasti, and M. Tonin, J. Math. Phys. 23, 839 (1982); L. Baulieu, Phys. Rep. 129, 1 (1985).

[8] C.M. Hull, B. Spence, and J.L. Vazquez-Bello, Nucl. Phys. B 348, 108 (1991).

[9] V.N. Shander, Functional analysis and its applications, 14, No. 2 (1980) 91 (in Russian).

[10] I.A. Batalin, K. Bering, and P.H. Damgaard, Nucl. Phys. B 515, 455 (1998); Phys. Lett. B 446, 175 (1999).

[11] E.S. Fradkin and G.A. Vilkovisky, Phys. Lett. B 55, 224 (1975); I.A. Batalin and G.A. Vilkovisky, Phys. Lett. B 69, 309 (1977); E.S. Fradkin and T.E. Fradkina, Phys. Lett. B 72, 343 (1978); I.A. Batalin and E.S. Fradkin, Phys. Lett. B 122, 157 (1983); For a review see: M. Henneaux, Phys. Rept. 126, 1 (1985).

[12] P.M. Lavrov, P.Yu. Moshin, and A.A. Reshetnyak, Mod. Phys. Lett. A 10, 2687 (1995); JETP Lett. 62, 780 (1995);

B. Geyer, P.M. Lavrov, and P.Yu. Moshin, Phys. Lett. B 463 , 188 (1999);

P.M. Lavrov and P.Yu. Moshin, Theor. Math. Phys. 126, 101 (2001).

[13] M. Alexandrov, M. Kontsevich, A. Schwarz, and O. Zaboronsky, Int. J. Mod. Phys. A 12, 1405 (1997).

[14] M. Grigoriev and P.H. Damgaard, Phys. Lett. B 474, 323 (2000).

[15] J.L. Koszul, Bull. Soc. Math. France 78, 5 (1950); A. Borel, Ann. Math. 57, 115 (1953);

J. Tate, Illinois J. Math. 1, 14 (1957);

J. Stasheff, Deformation theory and the Batalin-Vilkovisky master equation, Proceedings of the Conference on Deformation Theory (Ascona, Switzerland, 1996); q-alg/9702012; J.M.L. Fisch and M. Henneaux, Comm. Math. Phys. 128, 627 (1990).

[16] M. Henneaux and C. Teitelboim, Quantization of Gauge Systems (Princeton U.P., NJ 1992).
[17] D.M. Gitman and I.V. Tyutin, Quantization of Fields with Constraints, (Berlin and Heidelberg, Springer-Verlag, 1990).

[18] I.L. Buchbinder and S.M. Kuzenko, Ideas and Methods of Supersymmetry and Supergravity (Institute of Physics Publishing, Bristol \& Philadelphia, 1995)

[19] E. Noether, Nachr. Ges. Wiss. Goettingen, Math.-Phys. Kl. II (1918) 235;

M.A. Tavel, Transport Theory and Stat. Phys. 1, No. 3, 183 (1971).

[20] B.S. De Witt, Dynamical Theory of Groups and Fields (Gordon \& Breach, New York, 1965).

[21] F.A. Berezin, Introduction to Superanalysis (Eds. A.A. Kirillov and D. Leites, Reidel, Dordrecht, 1987).

[22] B. De Witt, Supermanifolds (2nd Edition, Cambridge University Press, Cambridge, 1992).

[23] I.A. Batalin and I.V. Tyutin, Mod. Phys. Lett. A 8, 3673 (1993); Int. J. Mod. Phys. A 9, 1707 (1994).

[24] A.A. Deriglazov, A.V. Galajinskii, and S.L. Lyachovich, Nucl. Phys. B 177, 282 (1996).

[25] An LSM of a general type, unlike an LSM of a special type, contains the operator $\partial_{\theta} \equiv \partial_{\theta}^{l}=d / d \theta$ in the structure functions of the gauge algebra.

[26] In the case of a nontrivial relation between the $t$ - and $\theta$ components of supertime $\chi$ by means of the operator $D=$ $\partial_{\theta}+\theta \partial_{t},[D, D]_{+}=2 \partial_{t}$, as the generator of the supergroup $J$, an adequate realization of $J$, whose quotient is the superspace $\mathcal{M}$, is made possible in terms of (non(super)commutative with each other) $\bar{J}$ and $P_{\text {ntriv }}=\exp (i \mu D)$ by analogy with the construction of a simple SUSY group.

[27] In the infinite-dimensional case, the concept of dimension has to be clarified; for a vector bundle $\mathcal{N} \rightarrow \widetilde{\mathcal{M}}$, it is formally understood that $\operatorname{dim} \mathcal{N}$ is the dimension of the fiber $\mathcal{F}_{p}^{\mathcal{N}}$ over an arbitrary $p \in \widetilde{\mathcal{M}}$.

[28] Here, the rank of a supermatrix is calculated with respect to the Grassmann parity $\varepsilon_{\tilde{J}}$ 